\title{
Evaluating the anharmonicity contributions to the molecular excited state internal conversion rates with finite temperature TD-DMRG
}

\author{
Yuanheng Wang, ${ }^{1}$ Jiajun Ren, ${ }^{1, \text { a) }}$ and Zhigang Shuai ${ }^{1, b}$ \\ MOE Key Laboratory of Organic OptoElectronics and Molecular Engineering, Department of Chemistry, Tsinghua University, \\ Beijing 100084, People's Republic of China
}

In this work, we propose a new method to calculate the molecular nonradiative electronic relaxation rates based on the numerically exact time-dependent density matrix renormalization group theory (TD-DMRG). This method could go beyond the existing frameworks under the harmonic approximation (HA) of the potential energy surface (PES) so that the anharmonic effect could be considered, which is of vital importance when the electronic energy gap is much larger than the vibrational frequency. We calculate the internal conversion (IC) rates in a two-mode model with Morse potential to investigate the validity of HA. We find that HA is unsatisfactory unless only the lowest several vibrational states of the lower electronic state are involved in the transition process when the adiabatic excitation energy is relatively low. As the excitation energy increases, HA first underestimates and then overestimates the IC rates when the excited state PES shifts towards the dissociative side of the ground state PES. On the contrary, HA slightly overestimates the IC rates when the excited state PES shifts towards the repulsive side. In both cases, higher temperature enlarges the error of HA. As a real example to demonstrate the effectiveness and scalability of the method, we calculate the IC rates of azulene from $S_{1}$ to $S_{0}$ on the ab initio anharmonic PES approximated by 1-mode representation. The calculated IC rates of azulene under HA are consistent with the analytically exact results. The rates on anharmonic PES are 30\%-40\% higher than the rates under HA.

\section{INTRODUCTION}

Nonradiative electronic relaxation (NRER) is an important process in the photophysics of molecular optoelectronic materials. It includes internal conversion process (IC) between the electronic states of the same spin manifold and intersystem crossing process (ISC) between the electronic states of different spin manifolds ${ }^{1}$ For organic photovoltaics and organic light-emitting system, the NRER processes from the excited state to the ground state is a harmful process which dissipates electronic energy into vibrational reservoirs and leads to the reduction of the energy conversion efficiency of the devices. Considering the important role of NRER in the molecular photophysical processes, how to calculate the rate of NRER theoretically has always been a hot topic. $2[9]$

Currently, the real-time nonadiabatic dynamics simulation and the rate theory relying on Fermi's golden rule (FGR) are the two main approaches to study the NRER process. Nonadiabatic dynamics directly simulate the nuclear motions over the coupled potential energy surfaces (PES) to obtain the real-time population on each electronic state. Though full-quantum dynamics methods have made great progress in recent years, it is still limited by the system size of complex molecules ${ }^{10-12]}$ Even if less accurate, nonadiabatic mixed quantum-classical dynamics (NA-MQC) methods provide a promising way to handle large systems $\left.{ }^{13}-15\right]$ One of the intriguing features of NA-MQC is that it could combine with the modern electronic structure calculation in an on-the-fly fashion to simulate ab initio dynamics without requiring a precomputed global PES which is necessary for most fullquantum wave-packet methods $\stackrel{16}{16}$ Recently, several semiclassical methods have also been extended to simulate the nona-

\footnotetext{
a)Electronic mail: renjj@ mail.tsinghua.edu.cn

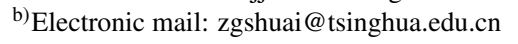

diabatic dynamics combined with the mapping strategy 17,20 It should be noted that in these methods the anharmonicity of the molecular PES is inherently considered. The main shortcoming of the real-time nonadiabatic dynamics methods to investigate the NRER process is that the accessible timescale is often limited to several picoseconds. Hence, they are suitable to describe the ultrafast transition process, such as transition through the conical intersection where the coupling between the electronic states is very strong. ${ }^{21}$ However, the NRER rates of a large portion of useful fluorescent molecules have timescales of nanosecond or even longer, ${ }^{22}$ far beyond what the current real-time nonadiabatic dynamics methods could accurately reach.

Complementary to the real-time simulation, in the regime where the coupling between the states is weak, the rate theory based on FGR has been successfully developed to describe the relatively slower processes. The study on this topic has a long history. Robinson and Frosch first outlined the harmonic oscillator approximation model to describe the NRER processes 50 years ago. ${ }^{2324}$ Afterwards, Lin established the framework using the displacement harmonic oscillator model to treat small polyatomic molecules with Duschinsky rotation effect (DRE) (mode-mixing effect) under the promoting mode approximation. ${ }^{325 \mid 26}$ In recent years, Shuai et al. have developed an analytical formalism called thermal vibration correlation function (TVCF) to calculate the NRER rate in the time domain. $5127-29]$ Under harmonic approximation (HA) of the initial and final electronic PES, this formalism could fully take DRE into consideration and gives the analytically exact transition rates. This method has been successfully used to calculate the NRER rate including IC and ISC processes of a lot of molecules at ab initio level ${ }^{2930}$ However, it is known that HA is only valid in the low energy regime around the equilibrium geometry, and the higher the energy, the stronger the anharmonic effect, especially for the floppy modes. Consequently, HA may not be reliable to describe the PES of the 
lower electronic state in the NRER process, because the large electronic excitation energy is dumped into the vibrations resulting in a relatively high vibrational quanta. Some former studies have attempted to investigate the anharmonic effect on the NRER rates of molecules in the FGR framework. Ianconescu and Pollak applied the semiclassical initial value representation method to study the IC rate in a two-mode model with Morse potential. ${ }^{31}$ They found that HA is mostly unsatisfactory in a wide parameter regime. Humeniuk et al. assessed the validity of HA for several coumarin dyes when predicting the fluorescence quantum yields in solution. ${ }^{32}$ They found that the accuracy of HA for the radiative decay rate is remarkable, while HA will underestimate the IC rates. Hence, HA will lead to an unreliable prediction of fluorescence quantum yield compared to the experiments. However, their method to deal with the Morse PES is based on the exact diagonalization and sum-of-states approach, which is not scalable to large systems. Though the aforementioned semiclassical method is scalable and seems promising in a model system, further benchmarking is still required to verify the universality. Therefore, it is important to develop a scalable and numerically exact method to calculate NRER rates beyond HA.

In this work, we propose to calculate the NRER rate with the numerically exact time-dependent density matrix renormalization group method (TD-DMRG) [33-36 In recent years, TD-DMRG has emerged as a powerful method to simulate large-scale full-quantum dynamics, 37,44 such as electronic spectroscopy of molecular aggregates, real-time internal conversion in pyrazine, carrier mobility in one-dimensional molecular crystal, etc. There are several advantages of TDDMRG compared to the other numerical methods: (i) The accuracy could be systematically improved by a single parameter; (ii) The Hamiltonian that can be handled is flexible once it could be represented in a sum-of-products (SOP) form $45[46$ and thus TD-DMRG could handle both model anharmonic PES and PES of real molecules after fitting or re-fitting to an

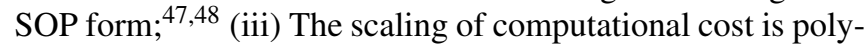
nomial with system size and thus it is scalable for polyatomic molecules; (iv) The time evolution of wavefunction (at zero temperature) and density matrix (at finite temperature) could be simulated in the same framework 4905 These advantages make TD-DMRG a suitable method to calculate the molecular NRER rates.

The remaining sections of this paper are arranged as follows: In section III THEORY, the Hamiltonian and method are described. In section III RESULTS \& DISCUSSIONS, firstly the IC rates of a two-mode model system with Morse potential are investigated to assess the validation of HA at different circumstances. Unlike the harmonic potential, the IC rate with the Morse potential is not analytically solvable. Secondly, as a real example to demonstrate the effectiveness and scalability of the method, the IC rates of azulene on the ab initio anharmonic PES approximated by 1-mode representation are calculated. The rates calculated under HA are also compared with the analytically exact results. Finally, the conclusion is presented in section IV CONCLUSION.

\section{THEORY}

\section{A. Hamiltonian and transition rate}

The molecular Hamiltonian of two uncoupled electronic states can be expressed as Eq. (1), where the mass-weighted coordinates $q_{l}$ are used. The potential energy operator is expanded by the two adiabatic electronic states $\left|\psi_{i}\right\rangle,\left|\psi_{f}\right\rangle$.

$$
\hat{H}_{0}=\sum_{l=1}^{N} \frac{\hat{p}_{l}^{2}}{2}+\left[\begin{array}{cc}
V_{f}\left(q_{1}, q_{2}, \cdots, q_{N}\right) & 0 \\
0 & V_{i}\left(q_{1}, q_{2}, \cdots, q_{N}\right)
\end{array}\right]
$$

$N$ is the total number of vibrational coordinates in the system. $V_{i / f}$ is the (semi-)global PES of the initial/final electronic state in the transition process. To set up the Hamiltonian for a specific molecule, the difficulty is how to obtain the PES $V_{i / f}$. Even nowadays, it is still a hard task to obtain a (semi-)global PES for polyatomic molecules with more than 20 atoms. For large systems, the high-order Taylor expansion of the PES at the equilibrium geometry is often used to calculate the anharmonic frequencies. ${ }^{5152}$ However, it is known that high-order Taylor expansion often has artificial "holes" on PES, which is disastrous for the variational approaches such as DMRG. The cut-high dimensional model representation (cut-HDMR) or called $n$-mode representation $(n-\mathrm{MR})$ method ${ }^{53 \mid 54}$ can partially solve this problem. Hence, we use $n$-MR to approximate PES of real molecule below. $n$-MR approximates the exact potential in a hierarchical manner. Eq. (2) shows 2-MR of PES.

$$
\begin{gathered}
V\left(q_{1}, q_{2}, \cdots, q_{N}\right)=V^{(0)}\left(\mathbf{q}^{\text {ref }}\right)+\sum_{i} V^{(1)}\left(q_{i} ; \mathbf{q}_{i}^{\text {ref }}\right) \\
+\sum_{i<j} V^{(2)}\left(q_{i}, q_{j} ; \mathbf{q}_{i j}^{\text {ref }}\right)+\cdots
\end{gathered}
$$

where

$$
\begin{gathered}
V^{(1)}\left(q_{i} ; \mathbf{q}_{i}^{\text {ref }}\right)=V\left(q_{i} ; \mathbf{q}_{i}^{\text {ref }}\right)-V^{(0)}\left(\mathbf{q}^{\text {ref }}\right) \\
V^{(2)}\left(q_{i}, q_{j} ; \mathbf{q}_{i j}^{\text {ref }}\right)=V\left(q_{i}, q_{j} ; \mathbf{q}_{i j}^{\text {ref }}\right)-V^{(1)}\left(q_{i} ; \mathbf{q}_{i}^{\text {ref }}\right) \\
-V^{(1)}\left(q_{j} ; \mathbf{q}_{j}^{\text {ref }}\right)-V^{(0)}\left(\mathbf{q}^{\text {ref }}\right)
\end{gathered}
$$

$V^{(0)}\left(\mathbf{q}^{\text {ref }}\right)$ is the energy at the reference point $\mathbf{q}^{\text {ref }}$, which is commonly chose at the equilibrium point. $V\left(q_{i} ; \mathbf{q}_{i}^{\text {ref }}\right)$ is the 1-dimensional (1-D) cut of the PES which only includes anharmonicity within single mode and $\left(q_{i} ; \mathbf{q}_{i}^{\text {ref }}\right)$ indicates that only $q_{i}$ is allowed to be different from the reference point. $V\left(q_{i}, q_{j} ; \mathbf{q}_{i j}^{\text {ref }}\right)$ is the 2-D cut of the PES which also includes mode-coupling. In $V^{(1)}$ and $V^{(2)}$, all the low order terms are excluded to avoid the double-counting. When $n=N$, the hierarchical expansion is exact. In practice, it is usually found that low order $n$-MR has already been accurate enough. One typical way to obtain $n$-MR is to compute the potential energy values on a set of grid points and then interpolate or fit functions accordingly. With the low order mode representation terms, it is convenient to convert them into the operators with an SOP format.55

Under HA, this difficulty to construct the (semi-)global PES is bypassed and only two normal mode analysis at the equilibrium geometry are required. The PES can be simplified with 
the normal coordinates.

$$
V_{i / f}=\sum_{l} \frac{1}{2} \omega_{i / f, l}^{2} q_{i / f, l}^{2}+V_{i / f}^{(0)}
$$

$\omega_{i / f, l}$ is the harmonic frequency of the $l$ th normal mode. The normal coordinates $q_{i / f}$ of the initial and final states are connected by the Duschinsky rotation matrix $S$ and the normalmode projected displacement $\Delta q$ as Eq. (6). The method to calculate these two parameters at ab initio level has been well established. 28156157

$$
q_{f, m}=\sum_{l} S_{m l} q_{i, l}-\Delta q_{f, m}
$$

The perturbation operator that couples the two electronic states is denoted as $\hat{H}_{1}$. In the IC process, $\hat{H}_{1}$ is the first order nonadiabatic coupling operator as Eq. (77).

$$
\hat{H}_{1}=\sum_{m}\left(\left\langle\psi_{i}\left|\hat{p}_{m}\right| \psi_{f}\right\rangle\left|\psi_{i}\right\rangle\left\langle\psi_{f}\right|+\text { h.c. }\right) \hat{p}_{m}
$$

In the ISC process, $\hat{H}_{1}$ is the spin-orbit coupling operator.

$$
\hat{H}_{1}=\left\langle\psi_{i}\left|\hat{V}_{\text {SOC }}\right| \psi_{f}\right\rangle\left|\psi_{i}\right\rangle\left\langle\psi_{f}\right|+\text { h.c. }
$$

When the coupling is weak, it is appropriate to calculate the transition rate between the two electronic states with FGR:

$$
W_{T}=\frac{2 \pi}{\hbar} \sum_{i, f} P_{i}\left|H_{1, i f}\right|^{2} \delta\left(E_{f}-E_{i}\right)
$$

$P_{i}$ is the Boltzmann distribution of the initial state $i$ at temperature $T$. We calculate $W_{T}$ in the time domain by Fourier transform of the Dirac function, the Eq. (10). Hence, the key to calculate the rate is to calculate the time correlation function (TCF) shown in Eq. (12), where $\beta=\left(k_{\mathrm{B}} T\right)^{-1}$ and $Z$ is the partition function.

$$
\begin{gathered}
\delta\left(E_{f}-E_{i}\right)=\frac{1}{2 \pi \hbar} \int_{-\infty}^{\infty} e^{-i\left(E_{f}-E_{i}\right) t / \hbar} d t \\
W_{T}=\frac{1}{\hbar^{2}} \int_{-\infty}^{\infty}\left\langle\hat{H}_{1}(t) \hat{H}_{1}\right\rangle_{T} d t \\
\left\langle\hat{H}_{1}(t) \hat{H}_{1}\right\rangle_{T}=\operatorname{Tr}\left(\frac{1}{Z} e^{-\beta \hat{H}_{0}} e^{i \hat{H}_{0} t / \hbar} \hat{H}_{1} e^{-i \hat{H}_{0} t / \hbar} \hat{H}_{1}\right)
\end{gathered}
$$

At $T=0$, TCF can be further simplified to Eq. (13),

$$
\left\langle\hat{H}_{1}(t) \hat{H}_{1}\right\rangle=e^{i E_{0} t / \hbar}\left\langle 0\left|\hat{H}_{1} e^{-i \hat{H}_{0} t / \hbar} \hat{H}_{1}\right| 0\right\rangle
$$

where $|0\rangle$ is the lowest eigenstate of the initial PES. In this work, we focus on the rate of IC process with nonadiabatic coupling operator as Eq. (7). But the rate of ISC process can be calculated in the same manner. For IC with the Condon approximation,

$$
\begin{aligned}
\left|H_{1, i f}\right|^{2} & =\left|\left\langle\phi_{i}\left|\left\langle\psi_{i}\left|\hat{H}_{1}\right| \psi_{f}\right\rangle\right| \phi_{f}\right\rangle\right|^{2}=\sum_{m, n} I_{m}^{*} I_{n} \\
I_{m} & =\left\langle\psi_{i}\left|\hat{p}_{m}\right| \psi_{f}\right\rangle\left\langle\phi_{i}\left|\hat{p}_{m}\right| \phi_{f}\right\rangle
\end{aligned}
$$

where $\phi_{i / f}$ is the vibrational wavefunction. From Eq. (14), we can find that $\left|H_{1, i f}\right|^{2}$ is a summation over two parts: diagonal terms with $n=m$ and off-diagonal terms with $n \neq m$. If the vibrational degrees of freedom (DoF) are uncoupled, $I_{m}$ can be further simplified as

$$
I_{m}=\left\langle\psi_{i}\left|\hat{p}_{m}\right| \psi_{f}\right\rangle\left\langle\chi_{i}\left(q_{m}\right)\left|\hat{p}_{m}\right| \chi_{f}\left(q_{m}\right)\right\rangle \prod_{l \neq m}\left\langle\chi_{i}\left(q_{l}\right) \mid \chi_{f}\left(q_{l}\right)\right\rangle,
$$

where $\chi\left(q_{m}\right)$ is the eigenstate of a single $\operatorname{DoF} q_{m}$.

\section{B. TD-DMRG method}

In TD-DMRG, the wavefunction ansatz is

$$
\begin{aligned}
|\Psi\rangle & =\sum_{\{\sigma\}} C_{\sigma_{1} \sigma_{2} \ldots \sigma_{N}}\left|\sigma_{1} \sigma_{2} \ldots \sigma_{N}\right\rangle \\
& =\sum_{\{a\},\{\sigma\}} A_{a_{1}}^{\sigma_{1}} A_{a_{1}, a_{2}}^{\sigma_{2}} \ldots A_{a_{N-1}}^{\sigma_{N}}\left|\sigma_{1} \sigma_{2} \ldots \sigma_{N}\right\rangle
\end{aligned}
$$

where $\left|\sigma_{i}\right\rangle$ is the orthonormal primitive basis set for each DoF. $N$ is the total number of DoFs in the system. As the full-rank coefficient $C_{\sigma_{1} \sigma_{2} \ldots \sigma_{N}}$ is approximated as the product of a chain of rank-3 matrix $A_{a_{i-1}, a_{i}}^{\sigma_{i}}$, this ansatz is called a matrix product state (MPS) $\stackrel{[50]}{T}$ The dimension of $a_{i}$ is called the (virtual) bond dimension, denoted as $M_{S}$. It is worth noting that the accuracy of an MPS can be systematically improved with $M_{S}$. The dimension of $\sigma_{i}$ is called the physical bond dimension, denoted as $d$. In this work, we use the simple harmonic oscillator basis to expand each DoF. If necessary, the discrete variable representation (DVR) $\sqrt{58}$ is used to approximate the matrix elements of potential energy operators such as the Morse-type operator. The details are given in the supplementary material. Similar to the wavefunction, a common operator $\hat{O}$ can also be represented in the matrix product form, called matrix product operator (MPO), as shown in Eq. (19).

$\hat{O}=\sum_{\{w\},\{\sigma\},\left\{\sigma^{\prime}\right\}} W_{w_{1}}^{\sigma_{1}^{\prime} \sigma_{1}} W_{w_{1}, w_{2}}^{\sigma_{2}^{\prime} \sigma_{2}} \ldots W_{w_{N-1}}^{\sigma_{N}^{\prime} \sigma_{N}}\left|\sigma_{1}^{\prime} \sigma_{2}^{\prime} \ldots \sigma_{N}^{\prime}\right\rangle\left\langle\sigma_{N} \sigma_{N-1} \ldots \sigma_{1}\right|$

With MPO, it is convenient to represent $\hat{O}|\Psi\rangle$ as another enlarged MPS with bond dimension $M_{O} M_{S}$.

$$
\hat{O}|\Psi\rangle=\sum_{\{w, a\},\left\{\sigma^{\prime}\right\}} A_{\{w, a\}_{1}}^{\prime \sigma_{1}^{\prime}} A_{\{w, a\}_{1},\{w, a\}_{2}}^{\prime \sigma_{2}^{\prime}} \ldots A_{\{w, a\}_{N-1}}^{\prime \sigma_{N}^{\prime}}\left|\sigma_{1}^{\prime} \sigma_{2}^{\prime} \ldots \sigma_{N}^{\prime}\right\rangle
$$

where

$$
A_{\{w, a\}_{i-1},\{w, a\}_{i}}^{\prime \sigma_{i}^{\prime}}=\sum_{\sigma_{i}} W_{w_{i-1}, w_{i}}^{\sigma_{i}^{\prime} \sigma_{i}} A_{a_{i-1}, a_{i}}^{\sigma_{i}}
$$

In Eq. (13), the initial state $|0\rangle$ at zero temperature can be obtained through the typical DMRG ground state algorithms by iteratively optimizing each local matrix $\mathrm{A} \cdot \frac{3450}{}$ At finite temperature, to obtain the thermal equilibrium density matrix $\rho_{\beta}=\frac{e^{-\beta H_{0}}}{Z(\beta)}$ for a canonical ensemble, the imaginary-time 
Schrödinger equation is integrated from $\tau=0$ to $\tau=\beta / 2$.

$$
-\frac{\partial}{\partial \tau} \rho(\tau)=\hat{H}_{0} \rho(\tau)
$$

The initial state $\rho(0)$ at infinitely high temperature $(\beta=0)$ is a locally maximally entangled state, which is easily represented as an MPO with $M_{O}=1$.

$$
\rho(0)=\prod_{i} \sum_{\sigma_{i}} \frac{1}{\sqrt{d}}\left|\sigma_{i}\right\rangle\left\langle\sigma_{i}\right|
$$

$\rho(\tau)$ is normalized with condition $\langle\langle\rho(\tau) \mid \rho(\tau)\rangle\rangle=$ $\operatorname{Tr}\left(\rho(\tau)^{\dagger} \rho(\tau)\right)=1$ after each step of time-evolution. Therefore, $\rho(\beta / 2)=e^{-\beta \hat{H}_{0} / 2} / \sqrt{Z(\beta)}=\rho_{\beta}^{1 / 2}$. Hence, the TCF in Eq. (12) can be re-expressed as:

$$
C(t)=\operatorname{Tr}\left(\rho_{\beta}^{1 / 2} e^{i \hat{H}_{0} t / \hbar} \hat{H}_{1} e^{-i \hat{H}_{0} t / \hbar} \hat{H}_{1} \rho_{\beta}^{1 / 2}\right)
$$

This method can equivalently be formulated according to the thermal field dynamics method, also known as the purification method by introducing an auxiliary space. 490

There are many time evolution schemes to propagate the wavefunction and density matrix according to the Schrödinger equation along the real-time or imaginary-time axes, and they are thoroughly compared in Ref. 59 and Ref. 60. In this work, we adopt the time-dependent variational principle-based evolution schemes. The variable-mean-field (VMF) scheme is used to propagate the wavefunction with matrix unfolding 61 and adaptive Dormand-Prince's 5/4 Runge-Kutta algorithm. The second-order projector-splitting scheme (PS) is used to propagate the density matrix for higher efficiency. Readers are referred to our former works for more details about the derivation and implementation ${ }^{60}$ The computational cost of a single time-step is $O\left(N\left(M_{S}^{2} M_{O}^{2} d^{2}+M_{S}^{3} M_{O} d+M_{S}^{3} d^{2}\right)\right)$ for the former and $O\left(N\left(M_{S}^{2} M_{O}^{2} d^{4}+M_{S}^{3} M_{O} d^{2}\right)\right)$ for the latter, which are both polynomial with system size. All the calculations in the next section are carried out with our in-house code Renormalizer.62

\section{RESULTS \& DISCUSSIONS}

\section{A. Two-mode model with Morse potential}

In this section, we adopt a minimal two-mode model with Morse potential as Ref. 31 to investigate the anharmonic effect on the internal conversion rate from the excited state to the ground state, in which PES of the ground state is characterized by two independent Morse potential along each vibrational DoF, while PES of the excited state is still harmonic (Typically, the excited state is prepared at low energies where a harmonic approximation is reasonable). In addition, there is no mode-mixing between the two PESs. The potential opera- tor is

$$
\begin{gathered}
V_{i}=V_{\mathrm{e}}=\sum_{l=1,2} \frac{1}{2} \omega_{\mathrm{e}, l}^{2} q_{\mathrm{e}, l}^{2}+V_{i}^{(0)} \\
V_{f}=V_{\mathrm{g}}=\sum_{l=1,2} D_{l}\left(1-e^{-\alpha_{l} q_{\mathrm{g}, l}}\right)^{2}+V_{f}^{(0)} \\
q_{\mathrm{e}, l}=q_{\mathrm{g}, l}-\Delta q_{l} \\
V_{i}^{(0)}-V_{f}^{(0)}=E_{\mathrm{ad}}
\end{gathered}
$$

where $E_{\mathrm{ad}}$ is the adiabatic excitation energy. The two parameters to define a Morse potential are the dissociation energy $D$ and the 'width' of the potential well $1 / \alpha$. A schematic diagram of the potential energy curve along one coordinate is shown in Fig. 1. A positive/negative $\Delta q$ represents that the excited state PES is shifted towards the dissociative/repulsive side of the ground state PES. Even though this model seems simple, unlike the harmonic potential, the internal conversion rate with the Morse potential cannot be calculated analytically. To construct the MPO for the system Hamiltonian, we use the symbolic method developed in our former work 46 to first construct the symbolic MPO and then expand every operator on the primitive basis to obtain a numerical one. The site ordering is another key aspect of a DMRG calculation. Although it was discussed to some extent in some former studies for vibronic models, $\stackrel{4143}{4}$ what is the optimal ordering is still unclear. In this calculation, the site ordering is $e, q_{1}, q_{2}$.

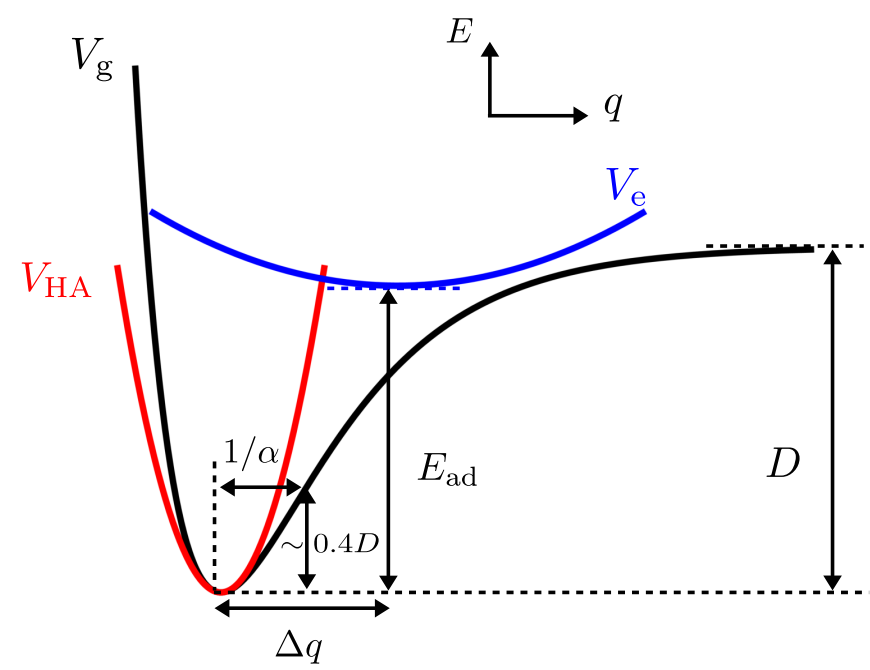

FIG. 1. A schematic diagram of the potential energy curve of the two-mode model along one coordinate. The black curve is the Morse potential $V_{g}=D\left(1-e^{-\alpha q}\right)^{2}$ of the ground state. The red curve is the harmonic approximation of the Morse potential at the equilibrium position. The blue curve is the harmonic potential of the excited state.

In order to compare with the results in Ref. 31, the same parameters are adopted here, $D_{1}=D_{2}=D=5.52 \mathrm{eV}$ and $\alpha_{1}=\alpha_{2}=\alpha=2.23 \mathrm{amu}^{-1 / 2} \AA^{-1}$ (0.0277 a.u.). Under HA, the harmonic frequency at the equilibrium position is $\omega_{\mathrm{g}, 1}=$ $\omega_{\mathrm{g}, 2}=\omega_{\mathrm{g}}=\sqrt{2 \alpha^{2} D}=3868 \mathrm{~cm}^{-1}$. The harmonic excited 
state PES has $\omega_{\mathrm{e}, 1}=\omega_{\mathrm{e}, 2}=\omega_{\mathrm{e}}=774 \mathrm{~cm}^{-1}$. In addition, the displacements are the same for the two DoFs $\Delta q_{1}=\Delta q_{2}=\Delta q$. The derivative coupling along each coordinate is set to be the same $\left\langle\psi_{\mathrm{e}}\left|\frac{\partial}{\partial q_{1}}\right| \psi_{\mathrm{g}}\right\rangle=\left\langle\psi_{\mathrm{e}}\left|\frac{\partial}{\partial q_{2}}\right| \psi_{\mathrm{g}}\right\rangle=C$. Hence, the generalized internal conversion rate is defined as $k_{\mathrm{ic}}=W_{T} / C^{2}$ using the constant $C^{2}$ as the unit. As in Ref. 31, TCF is multiplied by a Gaussian type broadening factor to make it converge after a finite period of time.

$$
W_{T}=\frac{1}{\hbar^{2}} \int_{-\infty}^{\infty}\left\langle\hat{H}_{1}(t) \hat{H}_{1}\right\rangle_{T} e^{-\frac{\sigma\left(E_{\mathrm{e}}\right)^{2} t^{2}}{2 \hbar^{2}}} d t
$$

$\sigma\left(E_{\mathrm{e}}\right)$ is chosen to represent the mean energy interval between the successive vibrational states on the ground state.

$$
\begin{gathered}
N\left(E_{\mathrm{e}}\right)=\operatorname{Tr}\left[\Theta\left(E_{\mathrm{e}}-\hat{H}_{\mathrm{g}}\right)\right] \approx \frac{1}{2}\left[\left(\frac{E_{\mathrm{e}}}{\hbar \omega_{\mathrm{g}}}\right)^{2}+\frac{E_{\mathrm{e}}}{\hbar \omega_{\mathrm{g}}}\right] \\
\sigma\left(E_{\mathrm{e}}\right)=\frac{\mathrm{d} E_{\mathrm{e}}}{\mathrm{d} N}
\end{gathered}
$$

where $\Theta$ is the Heaviside step function and $N\left(E_{\mathrm{e}}\right)$ is the number of quantum states below $E_{\mathrm{e}} . E_{\mathrm{e}}$ is the lowest energy of the excited vibronic state. As Ref. 31, the actual $\sigma\left(E_{\mathrm{e}}\right)$ used in all the calculations is 7 times the value defined in Eq. 30, 31. Since in the current model the two modes are not coupled or mixed, the formal propagator $e^{\hat{H}_{\mathrm{g} / \mathrm{e}} \tau}$ can be exactly represented as an MPO with $M_{O}=1$ (actually no matter what the system size is in this model), and the initial state $|0\rangle$ at zero temperature or $\rho(0)$ at finite temperature is also a Hartree product state with $M_{S}=1$. In addition, $\hat{H}_{1}$ could be represented as an MPO with $M_{O}=2$. Therefore, during the timeevolution, the time-dependent wavefunction in Eq. (13) and Eq. 24 could be exactly represented as an MPS with at least $M_{S}=2$ (The numerical results with different $M_{S}$ are shown in Fig. S1 in the supplementary material.). It should be mentioned that in Ref. 31, the Hamiltonian includes a momentum coupling term $\hat{p}_{1} \hat{p}_{2} / M$. Since this term is found to have only a minor effect on $k_{\text {ic }}$, it is neglected in the current work. In the subsequent numerical results, the time step is 8 a.u. (about $0.2 \mathrm{fs}$ ). The total simulation time is 240 a.u. to obtain the TCF using TD-DMRG and then $k_{\text {ic }}$ is calculated according to Eq. 29]. We note that in Ref. 31, only the diagonal terms $n=m$ of the summation in Eq. (14) are included to calculate $k_{\text {ic }}$ and the off-diagonal terms $n \neq m$ are all neglected. This approximation is similar to the widely known promoting mode approximation,,$\sqrt{3}$ which is valid in the case that only one mode called promoting mode has an appreciable derivative coupling and its displacement is approximately zero. However, considering that this approximation may not always be suitable for all systems, we include the off-diagonal terms when calculating the internal conversion rates.

First, we consider the zero temperature case in which the initial state is the lowest vibronic state of the excited state with zero vibrational quanta in each normal coordinate. With $\Delta q=0.7 / \alpha$ fixed, $k_{\text {ic }}$ with different $E_{\text {ad }}$ is shown in Fig. 2a in which only the diagonal terms in Eq. (14) are included. The results of TD-DMRG have already converged with physical bond dimension $d=60$ (the largest quanta of the harmonic oscillator basis) and are consistent with the results of
Ref. 31 by the semi-classical initial value representation approach. However, Fig. $2 \mathrm{~b}$ shows that the off-diagonal terms are also very important in this model, which increase $k_{\text {ic }}$ in some regimes and decrease it in the other regimes according to the different $E_{\text {ad }}$. This difference can be attributed to that the off-diagonal terms have different signs when the final vibronic state varies. Fig. 3 shows the relative size of the matrix elements of the off-diagonal terms to that of the diagonal terms $2 I_{1} I_{2} /\left(\left|I_{1}\right|^{2}+\left|I_{2}\right|^{2}\right)$, whose value is between -1 and 1 .

Since the Morse potential is asymmetrical unlike the harmonic potential, the direction of the relative displacement $\Delta q$ between the two PESs matters. Fig. 4 shows the 2D contour of the ratio of $k_{\mathrm{ic}}^{\mathrm{Morse}}$ on the Morse potential to $k_{\mathrm{ic}}^{\mathrm{HA}}$ on the harmonic potential with different displacement $\Delta q$ and adiabatic excitation energy $E_{\mathrm{ad}}$ at temperature $T=\omega_{\mathrm{g}} / 5$. At three representative displacements $\Delta q=0.7 / \alpha, 0 / \alpha$ and $-0.52 / \alpha, k_{\text {ic }}$ with different temperatures are shown in Fig. $4 b, 4 c$ and $4 d$, The convergence of the primitive basis set is shown in Fig. S2, S3 and S4 of the supplementary material. It is obvious that HA could give accurate results when $E_{\text {ad }}$ is relatively small $\left(E_{\mathrm{ad}} / D \sim 0\right)$. In this regime, only the vibronic state at the bottom of the ground state PES is involved in the transition process. For this low-energy state, HA is valid as expected. This situation would be encountered in the charge/energy transfer process between molecules of the same kind and ISC process in which the energies of the singlet and triplet state are very close such as the thermally activated delayed fluorescence system. ${ }^{63}$ However, higher energy and a larger positive displacement make the HA-valid regime much narrower. In the regimes that HA obviously fails, two trends can be found within the current model:

1. When the excited state PES shifts towards the dissociative side of the ground state PES $(\alpha \Delta q>0$, the top half of Fig. 4 a), HA will first underestimate $k_{\text {ic }}$ and then overestimate $k_{\text {ic }}$ as $E_{\text {ad }}$ increases. In addition, $k_{\text {ic }}$ with the Morse potential drops much rapidly as $E_{\mathrm{ad}}$ increases compared to that with harmonic potential once the peak is passed (Fig. 4b. 4c).

2. When the excited state PES shifts towards the repulsive side of the ground state PES $(\alpha \Delta q<0$, the bottom half of the Fig. 4 ), HA slightly overestimates $k_{\text {ic }}$ (Fig.4d).

To examine the generality of the trends described above, we also calculate $k_{\text {ic }}$ with $D^{\prime}=D, \alpha^{\prime}=2 / 3 \alpha$ and $D^{\prime}=4 / 9 D$, $\alpha^{\prime}=\alpha$. The similar 2D contours as Fig. $4 \mathrm{a}$ are shown in Fig. S5 of the supplementary material. The trends are qualitatively the same. Besides these two trends, in both cases, the higher the temperature, the greater the error of HA.

Two fundamental differences between the vibrational wavefunctions of Morse potential $\chi^{\text {Morse }}$ and harmonic potential $\chi^{\mathrm{HA}}$ with the same quantum number $n$ may explain the two trends. First, the amplitude of $\chi^{\text {Morse }}$ is larger than $\chi^{\mathrm{HA}}$ on the dissociative side, while smaller on the repulsive side, as shown in the middle panels of Fig. $5 \mathrm{a}(n=3)$ and Fig. $5 \mathrm{~b}$ $(n=10)$. Second, by comparing these two panels, $\chi^{\text {Morse }}$ spreads very fast to the dissociative side as the quantum number increases, while $\chi^{\mathrm{HA}}$ with the same quantum number is 


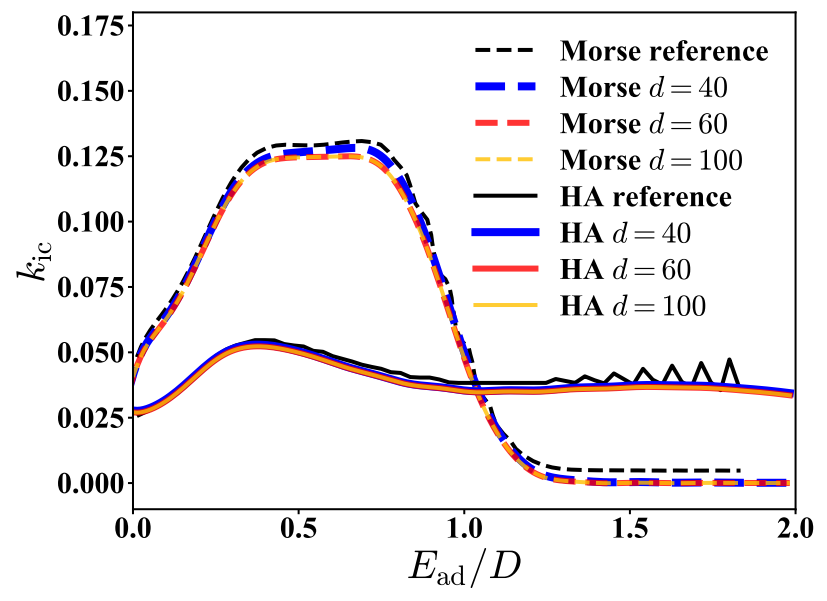

(a)

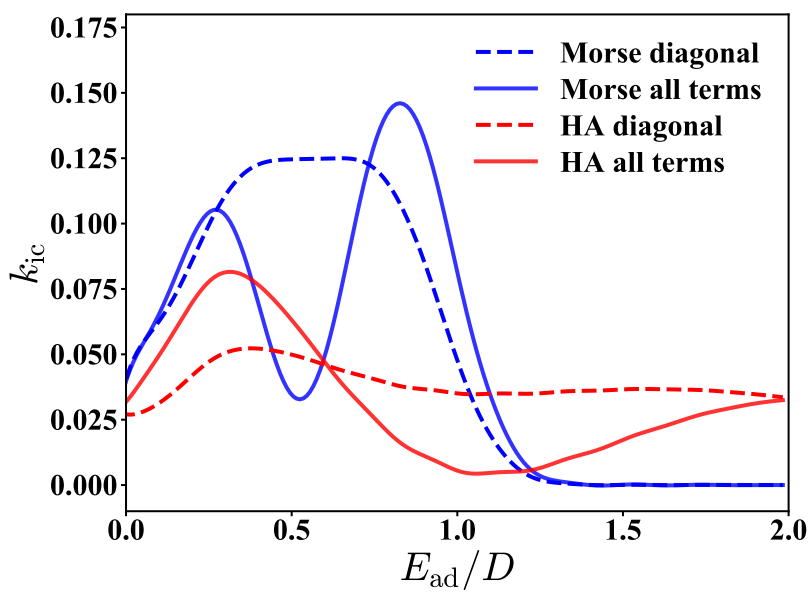

(b)

FIG. 2. a) The dependence of $k_{\text {ic }}$ on the adiabatic excitation energy $E_{\mathrm{ad}} / D$ at zero temperature calculated by TD-DMRG with different sizes of primitive basis sets. Only the diagonal terms in Eq. (14) are included. The results in Ref. 31 are also plotted for comparison (black line). b) $k_{\text {ic }}$ with or without the off-diagonal terms in Eq. 14 calculated by TD-DMRG with $d=60$. (The displacement is $\Delta q=0.7 / \alpha$. The virtual bond dimension used is $M_{S}=4$. Morse: full treatment of the anharmonic Morse PES. HA: harmonic approximation of the Morse potential. )

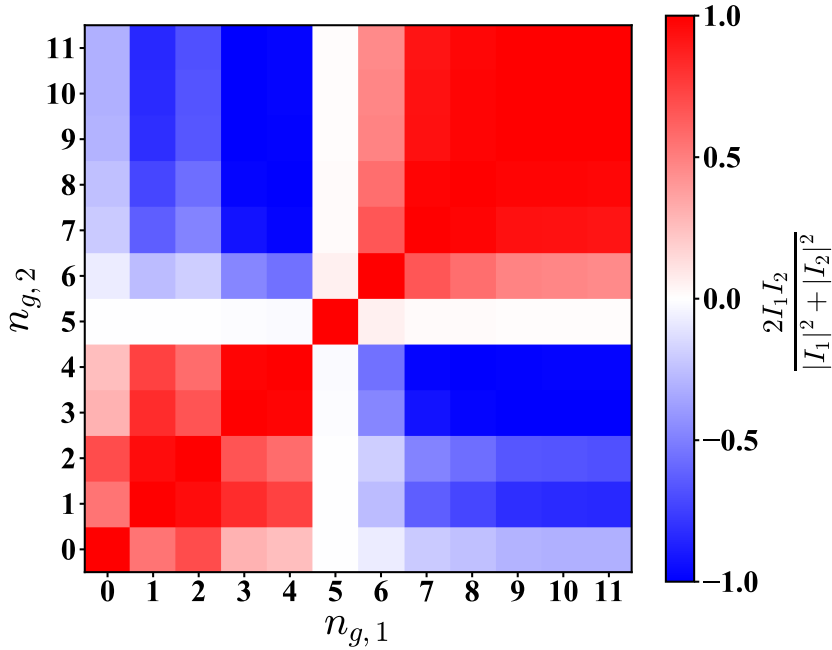

FIG. 3. The relative size of the matrix elements of the off-diagonal terms to that of the diagonal terms $2 I_{1} I_{2} /\left(\left|I_{1}\right|^{2}+\left|I_{2}\right|^{2}\right)$ defined in Eq. (14) and Eq. (15) at zero temperature. The vibrational wavefunction $\phi_{\mathrm{g}}\left(q_{1}, q_{2}\right)=\chi_{n_{\mathrm{g}, 1}}^{\mathrm{HA}}\left(q_{1}\right) \chi_{n_{\mathrm{g}, 2}}^{\mathrm{HA}}\left(q_{2}\right)$ of the final state is characterized by two quantum number $-n_{\mathrm{g}, 1}$ and $n_{\mathrm{g}, 2}$, which are both ranging from 0 to 11 .

relatively localized. Consequently, when $\alpha \Delta q>0$ and the quantum number of the final vibrational state is small $\left(E_{\mathrm{ad}}\right.$ is small), the larger amplitude of $\chi^{\text {Morse }}$ in the region of the initial vibrational wavefunction $\chi_{\mathrm{e}}(n=0)$ will result in a larger overlap $S_{\mathrm{g}, \mathrm{e}}^{\mathrm{M}} \mathrm{rse}$ and thus a larger Franck-Condon (FC) factor as shown in the upper panel of Fig. $5 \mathrm{a}(n=3)$ and so is the transition rate $k_{\mathrm{ic}}$. As the quantum number increases, $\chi^{\text {Morse }}$ quickly spreads to the dissociative side and the amplitude of $\chi^{\text {Morse }}$ in the region of $\chi_{\mathrm{e}}$ decays much more rapidly once the large head of $\chi^{\text {Morse }}$ crosses $\chi_{\mathrm{e}}$ compared to the more localized $\chi^{\mathrm{HA}}$, resulting in a smaller FC factor as shown in the upper panel of Fig. $5 \mathrm{~b}(n=10)$. Quantitatively, $S_{\mathrm{g}, \mathrm{e}}^{\mathrm{Morse}} \mathrm{de}-$ creases from 0.4 to 0.025 while $S_{\mathrm{g}, \mathrm{e}}^{\mathrm{HA}}$ only decreases from 0.3 to 0.2 when the quantum number increases from 3 to 10 . In addition, $\chi^{\text {Morse }}$ has more nodes than $\chi^{\mathrm{HA}}$ with similar excitation energy, leading to a more serious phase cancellation when calculating the overlap. On the repulsive side, though $\chi^{\text {Morse }}$ is also localized, the amplitude of $\chi^{\text {Morse }}$ is smaller than that of $\chi^{\mathrm{HA}}$, resulting in a smaller overlap as shown in the lower panel of Fig. 5a and Fig. 5b To understand the temperature effect, Fig. 6 shows that the square of matrix element $\left\langle\chi_{\mathrm{e}}(q)\left|\frac{\partial}{\partial q}\right| \chi_{\mathrm{g}}(q)\right\rangle$ in Eq. (16) (playing the role as a prefactor of the FC factor) is relatively larger for initial state with higher vibrational quanta $n_{\mathrm{e}}$. Therefore, when the thermally populated initial states with higher vibrational quanta get involved with the temperature, the error of HA is significantly larger.

To show the computational complexity of the proposed method with system size, we increase the system size from 2 to 20. Please see supplementary materials for details. Fig. S6 shows that the computational cost is linearly dependent on the system size for the current uncoupled model without modemixing. If the bi-mode coupling term $\sum_{l<k} \Gamma q_{l}^{2} q_{k}^{2}$ is considered, both the size of MPO and the required $M_{S}$ will increase. Hence, the computational cost almost grows cubically with system size. 


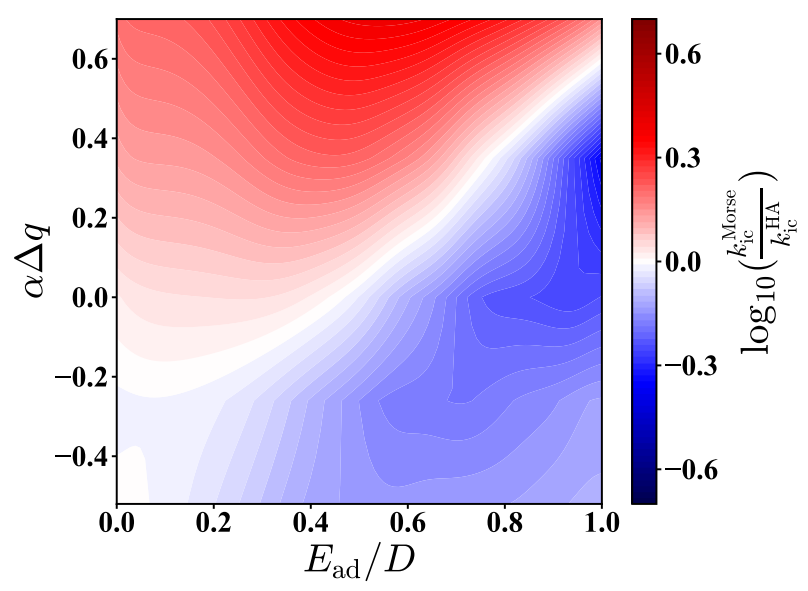

(a)

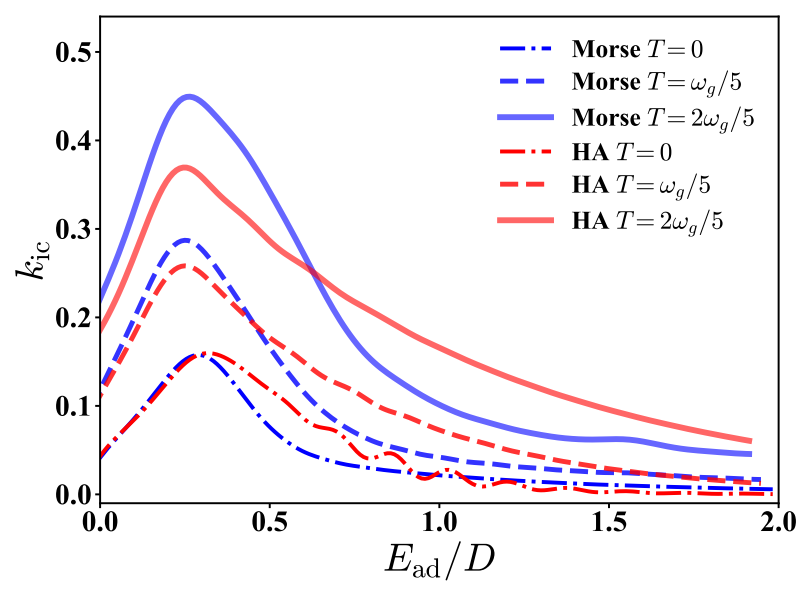

(c)

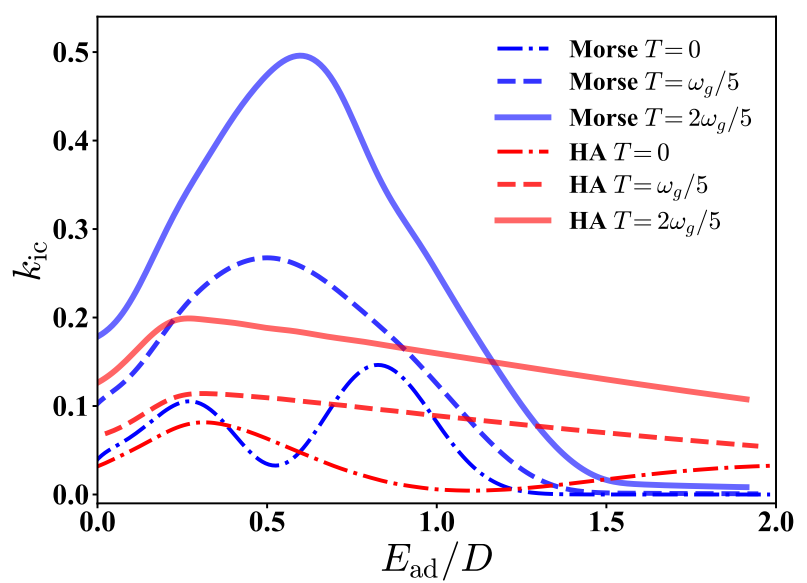

(b)

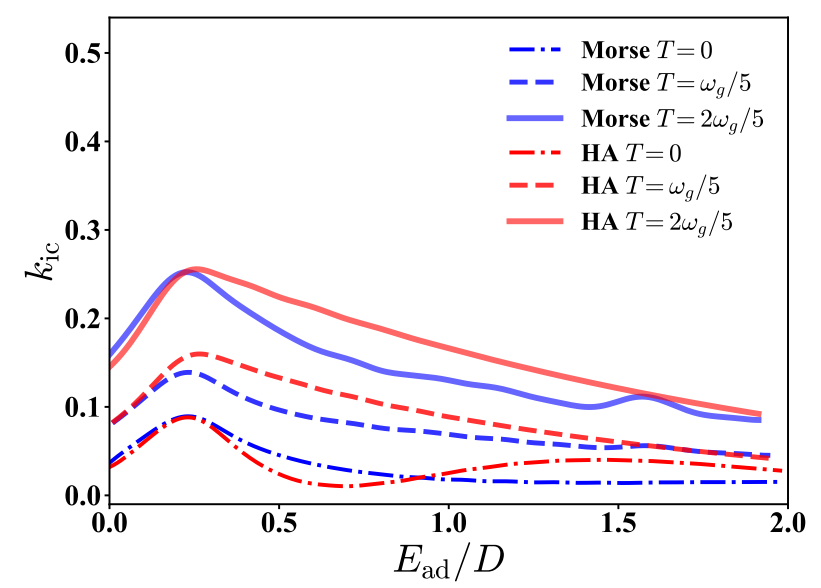

(d)

FIG. 4. a) The ratio of the internal conversion rate on the Morse potential with respect to that under HA with different displacements and adiabatic excitation energies. The temperature is $\left.T=\omega_{\mathrm{g}} / 5 . \mathrm{b}\right)-\mathrm{d}$ ) The dependence of $k_{\mathrm{ic}}$ on the adiabatic excitation energy calculated by TD-DMRG with different displacements b) $\Delta q=0.7 / \alpha, \mathrm{c}) \Delta q=0 / \alpha$ and d) $\Delta q=-0.52 / \alpha$, at different temperatures $\left(T=0, \omega_{\mathrm{g}} / 5,2 \omega_{\mathrm{g}} / 5\right)$, with or without HA. The physical and virtual bond dimensions in all these calculations are $d=100$ and $M_{S}=4$. The comparison of the results with different $d$ is shown in the supplementary material.

\section{B. IC rate of azulene}

The proposed method can be applied to the real molecules if the PES is available. As an example to demonstrate the effectiveness and scalability of the method in real molecules, in this section we calculate the internal conversion rate of azulene from the $S_{1}$ state to $S_{0}$ state. Azulene has often been used as a prototypical system to benchmark new methods. ${ }^{[7 \mid 28}$ Here, two types of PES are considered: (i) the harmonic PES expanded around the respective equilibrium geometry of ground state and excited state. (ii) the ground state PES is approximated by 1-MR along each normal coordinate (the excited state is still considered to be harmonic). As introduced above, 1-MR includes the anharmonicity of 1-D cut of the PES along each coordinate. The single point energy, equilibrium geome- try, and normal mode analysis of the ground state and excited state of azulene are calculated by density functional theory (DFT) and time-dependent DFT at B3LYP/6-31G(d) level in Gaussian $16 \cdot{ }^{64}$ The number of normal modes of azulene is 48 . The Duschinsky rotation matrix $S$ and normal mode projected displacement $\Delta q$ as in Eq. (6) are calculated by MOMAP.65 The 1-MR PES is constructed by the adaptive density-guided approach (ADGA) implemented in MidasCpp 66 developed by Christiansen et al ${ }^{67}$ A total of 741 ab initio points are calculated and the 1-D cut of PES is fitted with polynomial functions up to 12 th order. The 48 1-D PES cuts are shown in Fig. S9-S12 of the supplementary material. It is clear to see that azulene is a semi-rigid molecule with a well-defined minimum corresponding to the equilibrium geometry. In the TD-DMRG calculations, the coordinates used are the normal 


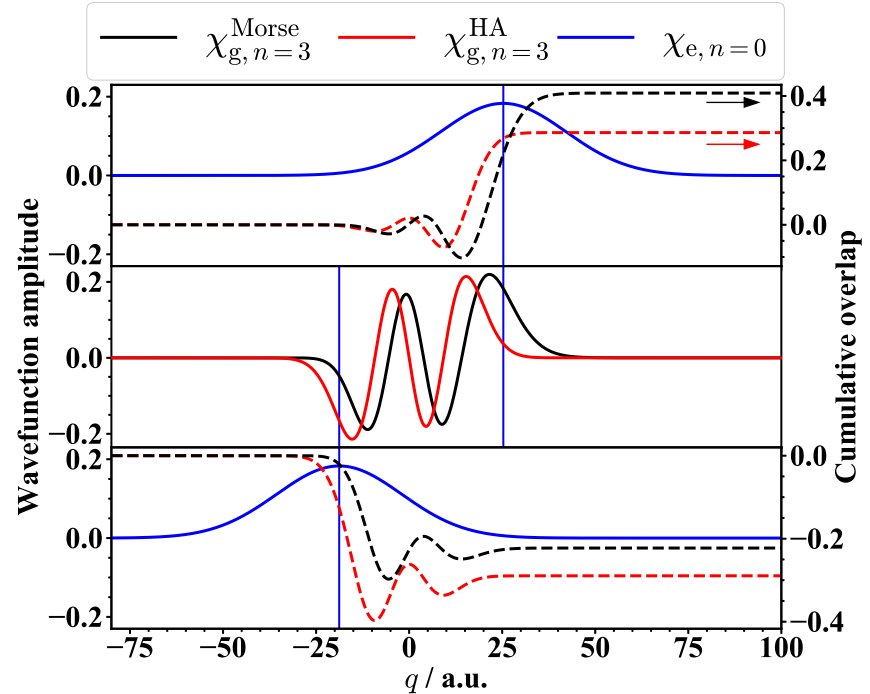

(a)

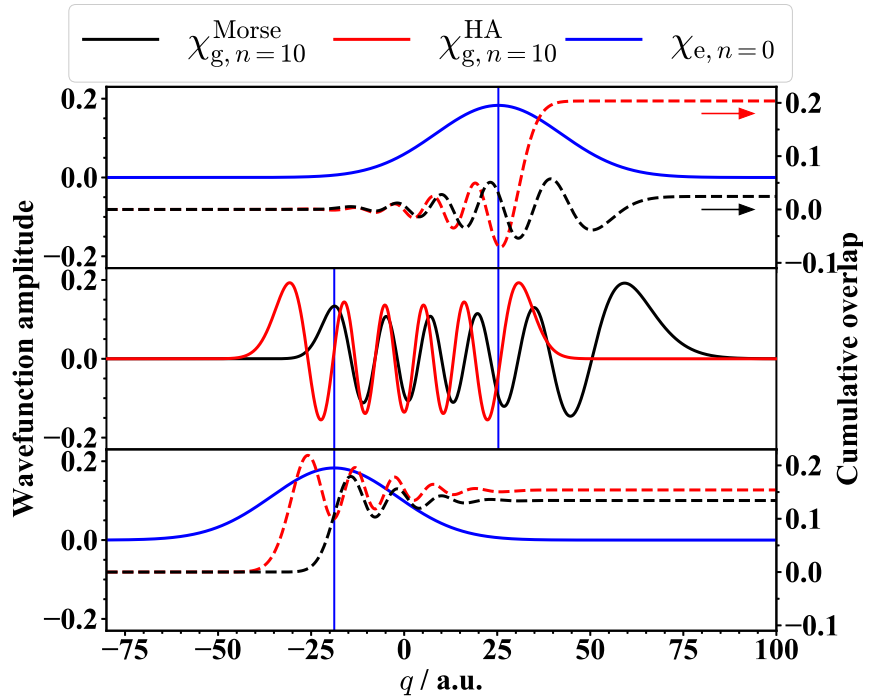

(b)

FIG. 5. a) Middle panel: the vibrational wavefunction of the ground state with the quantum number $n=3$ on the Morse potential (solid black) and approximated harmonic potential under HA (solid red). Upper panel: the lowest vibrational wavefunction of the excited state with $\Delta q=0.7 / \alpha$ (solid blue) and the cumulative overlap between the initial and final wavefunctions (dashed black and red lines). Lower panel: same as the upper panel but with $\Delta q=-0.52 / \alpha$. b) same as (a) but the quantum number of vibrational state of the electronic ground state is $n=10$.

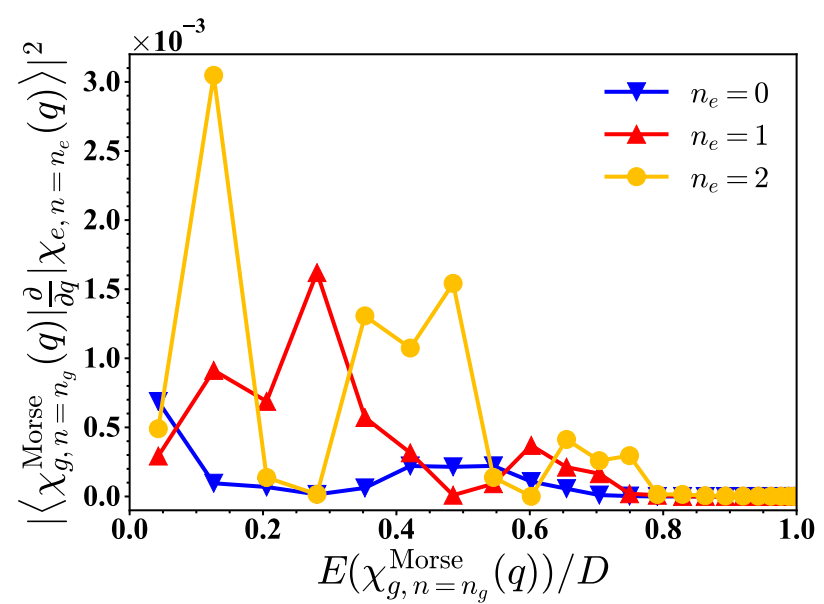

(a)

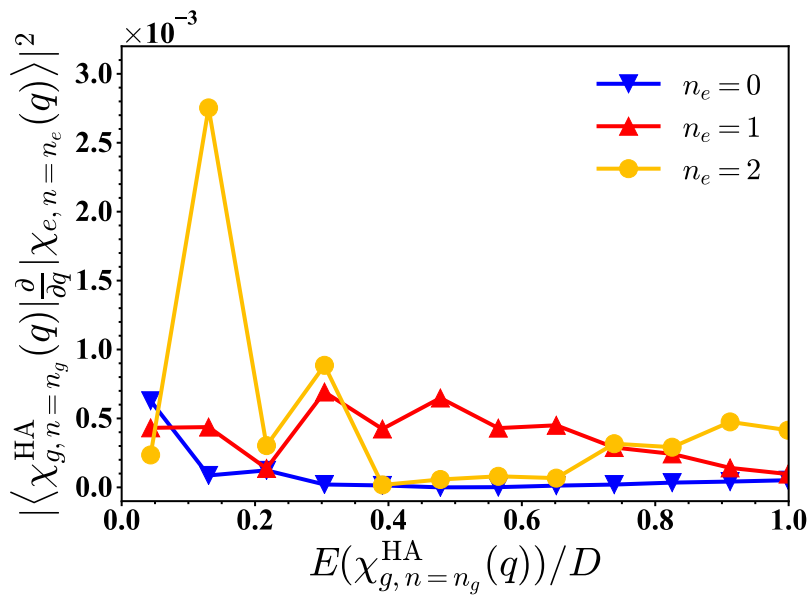

(b)

FIG. 6. The square of the matrix elements $\left\langle\chi_{\mathrm{e}}(q)\left|\frac{\partial}{\partial q}\right| \chi_{\mathrm{g}}(q)\right\rangle$ between different initial vibrational states of electronic excited state (distinguished by vibrational quanta $n_{\mathrm{e}}$ ) and series of final vibrational states of electronic ground state (distinguished by the energy $E\left(\chi_{\mathrm{g}, n}\right)$ ) on a) Morse potential, b) harmonic potential under HA. Each triangle, inverted triangle or circle denotes a state with lines as a guide to the eye. The results are calculated through exact diagonalization. The displacement is $\Delta q=0.7 / \alpha$.

coordinates of the ground state. They are arranged in ascending order of harmonic frequency. The site of the electronic DoF is put to the middle of the chain. The time step is $0.25 \mathrm{fs}$ and the total time of simulation is $425 \mathrm{fs}$. The primitive basis for each DoF is the harmonic oscillator basis up to 20 quanta. A Lorentzian broadening factor $100 \mathrm{~cm}^{-1}$ is applied to make the time-integration of TCF converge.

For the harmonic PES, the TVCF method ${ }^{527}$ (implemented in MOMAF ${ }^{65}$ ) is analytically exact and thus serves as a reference here. For comparison, the same time step and total evolution time are used in TVCF. The TCF $C(t)$ in Eq. (24) calculated by TVCF and TD-DMRG with different bond di- 
mension $M_{S}$ are shown in Fig. 7 The results with $M_{S}=2$ (blue dashed line) deviate from the exact value after $40 \mathrm{fs}$ and thus is not accurate enough to calculate $k_{\text {ic }}$ (See Table. I). The results with $M_{S}=20$ (red dashed line) are consistent with the exact values at the resolution scale shown in Fig. 7. The transition rates $k_{\text {ic }}$ are listed in Table. I The analytically exact value is $2.17 \times 10^{10} \mathrm{~s}^{-1}$ at $0 \mathrm{~K}$ and $2.44 \times 10^{10} \mathrm{~s}^{-1}$ at $300 \mathrm{~K}$. The results of TD-DMRG converge very fast with $M_{S}$ and $M_{S}=20$ could obtain a quantitatively accurate rate $-2.11 \times 10^{10} \mathrm{~s}^{-1}$ at $0 \mathrm{~K}$ and $2.32 \times 10^{10} \mathrm{~s}^{-1}$ at $300 \mathrm{~K}$. The computational wall-clock time for the whole simulation with $M_{S}=20$ is 35 minutes at $0 \mathrm{~K}$ and 6 hours 33 minutes at $300 \mathrm{~K}$ with 4 Intel Xeon Gold 5115 CPU cores and 1 NVIDIA V100 GPU.

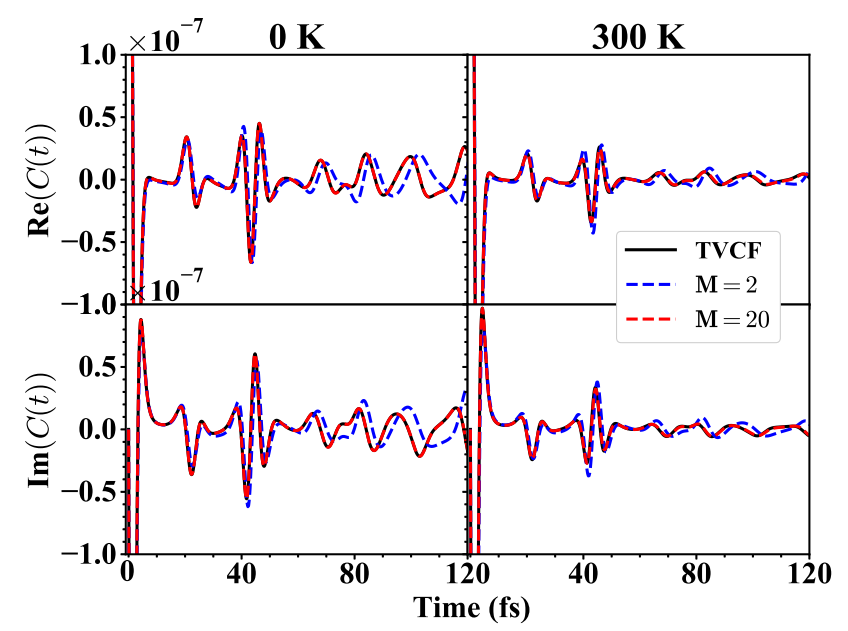

FIG. 7. The real and imaginary part of the time correlation function $C(t)$ at $T=0 \mathrm{~K}$ and $T=300 \mathrm{~K}$ calculated by the analytically exact TVCF method implemented in MOMAF ${ }^{65}$ (black solid line) and TDDMRG with bond dimension $M_{S}=2$ (blue dashed line) and $M_{S}=20$ (red dashed line).

Though on the harmonic potential TD-DMRG method is definitely much more expensive than the TVCF method, TDDMRG could go beyond HA and the cost is not expected to increase too much depending on the specific form of the anharmonic PES. For the anharmonic PES of azulene approximated by $1-\mathrm{MR}$, the results with different $M_{S}$ are also listed in Table. I. The results of TD-DMRG still converge very fast with $M_{S}$ and at $M_{S}=60, k_{\text {ic }}$ is $2.89 \times 10^{10} \mathrm{~s}^{-1}$ at $0 \mathrm{~K}$ and $3.37 \times 10^{10} \mathrm{~s}^{-1}$ at $300 \mathrm{~K}$, which is roughly $30 \%-40 \%$ higher than the results of harmonic PES. The results are consistent with the findings in the two-mode model above. For the multimode molecule in the weak coupling limit with all the HuangRhys factor $S_{i}<1$ (Fig. S13 shows $S_{i}$ of azulene), it has been known that the most probable final states prefer to simultaneously excite several vibrational modes to accept the electronic energy together rather than excite only one mode to very high energy level. ${ }^{68}$ Therefore, for each mode, the energy received is in the small to medium regime in which the rate on the Morse potential is mainly larger than that on the harmonic potential (Fig. 4). The computational wall-clock time for the whole simulation with $M_{S}=20$ is 26 minutes at $0 \mathrm{~K}$ and 7 hours 1 minute at $300 \mathrm{~K}$, which is similar to the harmonic case. This is because the modes are still independent in 1-MR PES and thus the bond dimension $M_{O}$ of MPO does not change and the required $M_{S}$ for the same accuracy is also roughly the same from Table. II. When 2-MR PES is considered, we expect that the cost spent in TD-DMRG will increase because both $M_{S}$ and $M_{O}$ will increase but still affordable. However, to construct the 2-MR PES for azulene needs at least 100,000 single point ab initio calculations (assuming 10 grids on each coordinate), which will in turn become the bottleneck of the whole calculation.

\section{CONCLUSION}

In this work, we propose to use TD-DMRG to calculate the rate of molecular nonradiative electronic relaxation process based on Fermi's golden rule. Firstly, we calculate the internal conversion rate of a two-mode model system with Morse potential and assess the validity of harmonic approximation. We emphasize that the off-diagonal terms neglected in the former studies are also important to the transition rate and the harmonic approximation is unsatisfactory in a large parameter regime unless only the lowest several vibrational states of the lower electronic state are involved in the transition process when the adiabatic excitation energy is relatively low. Since the Morse potential is asymmetrical, the error of the harmonic approximation strongly depends on the direction of the shift of the excited state potential energy surface with respect to the ground state. When $\alpha \Delta q>0$, the harmonic approximation will first underestimate the IC rate and then overestimate it as the excitation energy increases. This is due to that the amplitude of the wavefunction on the Morse potential is larger than that of the harmonic potential in the dissociative side but the wavefunction spreads quickly with energy while the harmonic wavefunction is much more localized. Hence, the Franck-Condon factor between the initial and final states on the Morse potential is first larger and then smaller than that under harmonic approximation. When $\alpha \Delta q<0$, the harmonic approximation will slightly overestimate the IC rate because the wavefunction on the Morse potential is also localized on this side but the amplitude is smaller. Moreover, higher temperature will enlarge the error of harmonic approximation. Secondly, we calculate the internal conversion rate of azulene. Under harmonic approximation, the results are consistent with the analytically exact results calculated by the thermal vibration correlation function method. On the anharmonic PES approximated by one-mode representation, the results are $30 \%-40 \%$ higher than that on harmonic PES, indicating that in this semi-rigid system the anharmonic effect on IC process is not very strong. The computational cost is roughly the same compared to harmonic case, which demonstrates the effectiveness and scalability of the current method to be applied to large polyatomic molecules. It should be mentioned that though we focus on the rate of internal conversion process in the numerical examples in this work, the same approach could also be used in the calculation of the in- 
TABLE I. The internal conversion rate $k_{\text {ic }}$ of azulene from the $S_{1}$ state to the $S_{0}$ state with harmonic PES and with anharmonic 1-MR PES calculated by TD-DMRG with different bond dimension $M_{S}$. The analytically exact results with harmonic PES calculated by TVCF are also listed.

\begin{tabular}{cccccc}
\hline \multirow{2}{*}{ Method } & & \multicolumn{2}{c}{$k_{\text {ic }}\left(\times 10^{10} \mathrm{~s}^{-1}\right)$ at $0 \mathrm{~K}$} & \multicolumn{2}{c}{$k_{\mathrm{ic}}\left(\times 10^{10} \mathrm{~s}^{-1}\right)$ at $300 \mathrm{~K}$} \\
$1-\mathrm{MR}$
\end{tabular}

tersystem crossing rates. Finally, floppy molecules, such as the aggregation-induced emission systems, $\underset{69}{ }$ may have a significant anharmonic effect on the IC process, thus applying the current TD-DMRG method to these systems with ab initio anharmonic potential energy surface is worth further study.

\section{SUPPLEMENTARY MATERIAL}

See the supplementary material for the internal conversion rates $k_{\mathrm{ic}}$ of the two-mode model calculated by TD-DMRG with different virtual bond dimension $M_{S}$, physical bond dimension $d$, and different Morse potential parameters. The 1-D cuts of azulene ground state PES can also be found.

\section{ACKNOWLEDGMENTS}

This work is supported by the National Natural Science Foundation of China (NSFC) through the project "Science CEnter for Luminescence from Molecular Aggregates (SCELMA)" Grant Number 21788102, as well as by the Ministry of Science and Technology of China through the National Key R\&D Plan Grant Number 2017YFA0204501. J.R. is also supported by the NSFC via Grant Number 22003029.

\section{DATA AVAILABILITY}

The data that support the findings of this study are available from the corresponding author upon reasonable request.

${ }^{1}$ N. J. Turro, Modern molecular photochemistry (University science books, 1991).

${ }^{2}$ R. Englman and J. Jortner, "The energy gap law for radiationless transitions in large molecules," Mol. Phys. 18, 145-164 (1970).

${ }^{3}$ S. Lin, C. Chang, K. Liang, R. Chang, J. Zhang, T. Yang, M. Hayashi, Y. Shiu, and F. Hsu, "Ultrafast dynamics and spectroscopy of bacterial photosynthetic reaction centers," Adv. Chem. Phys. 121, 1-88 (2002).

${ }^{4}$ Q. Shi and E. Geva, "Nonradiative electronic relaxation rate constants from approximations based on linearizing the path-integral forward- backward action," J. Phys. Chem. A 108, 6109-6116 (2004).

${ }^{5}$ Q. Peng, Y. Yi, Z. Shuai, and J. Shao, "Excited state radiationless decay process with duschinsky rotation effect: formalism and implementation," J. Chem. Phys. 126, 114302 (2007)
${ }^{6}$ R. Ianconescu, J. Tatchen, and E. Pollak, "On-the-fly semiclassical study of internal conversion rates of formaldehyde," J. Chem. Phys. 139, 154311 (2013).

${ }^{7}$ S. Banerjee, A. Baiardi, J. Bloino, and V. Barone, "Temperature dependence of radiative and nonradiative rates from time-dependent correlation function methods," J. Chem. Theory Comput. 12, 774-786 (2016).

${ }^{8} \mathrm{X}$. Sun and E. Geva, "Equilibrium fermi's golden rule charge transfer rate constants in the condensed phase: The linearized semiclassical method vs classical marcus theory," J. Phys. Chem. A 120, 2976-2990 (2016).

${ }^{9}$ A. Celestino and A. Eisfeld, "Tuning nonradiative lifetimes via molecular aggregation,” J. Phys. Chem. A 121, 5948-5953 (2017).

${ }^{10}$ H.-D. Meyer, "Studying molecular quantum dynamics with the multiconfiguration time-dependent hartree method," Wiley Interdiscip Rev Comput Mol Sci 2, 351-374 (2012).

${ }^{11}$ G. Richings, I. Polyak, K. Spinlove, G. Worth, I. Burghardt, and B. Lasorne, "Quantum dynamics simulations using gaussian wavepackets: the vmcg method," Int. Rev. Phys. Chem. 34, 269-308 (2015).

${ }^{12}$ B. F. Curchod and T. J. Martínez, "Ab initio nonadiabatic quantum molecular dynamics," Chem. Rev. 118, 3305-3336 (2018).

${ }^{13} \mathrm{G}$. Cui and W. Thiel, "Generalized trajectory surface-hopping method for internal conversion and intersystem crossing," J. Chem. Phys. 141, 124101 (2014).

${ }^{14} \mathrm{R}$. Crespo-Otero and M. Barbatti, "Recent advances and perspectives on nonadiabatic mixed quantum-classical dynamics," Chem. Rev. 118, 7026$7068(2018)$

${ }^{15}$ L. Wang, J. Qiu, X. Bai, and J. Xu, "Surface hopping methods for nonadiabatic dynamics in extended systems," Wiley Interdiscip Rev Comput Mol Sci 10, e1435 (2020).

${ }^{16}$ M. H. Beck, A. Jäckle, G. A. Worth, and H.-D. Meyer, "The multiconfiguration time-dependent hartree (mctdh) method: a highly efficient algorithm for propagating wavepackets," Phys. Rep. 324, 1-105 (2000).

${ }^{17} \mathrm{~S}$. N. Chowdhury and P. Huo, "Coherent state mapping ring polymer molecular dynamics for non-adiabatic quantum propagations," J. Chem. Phys. 147, 214109 (2017)

${ }^{18}$ X. Gao, Y. Lai, and E. Geva, "Simulating absorption spectra of multiexcitonic systems via quasiclassical mapping hamiltonian methods," J. Chem. Theory Comput. 16, 6465-6480 (2020).

${ }^{19} \mathrm{X}$. Gao and E. Geva, "A nonperturbative methodology for simulating multidimensional spectra of multiexcitonic molecular systems via quasiclassical mapping hamiltonian methods," J. Chem. Theory Comput. 16, 6491-6502 (2020).

${ }^{20}$ J. Zheng, J. Peng, Y. Xie, Y. Long, X. Ning, and Z. Lan, "Study of the exciton dynamics in perylene bisimide (pbi) aggregates with symmetrical quasiclassical dynamics based on the meyer-miller mapping hamiltonian," Phys. Chem. Chem. Phys. 22, 18192-18204 (2020).

${ }^{21}$ A. Toniolo, S. Olsen, L. Manohar, and T. J. Martinez, "Conical intersection dynamics in solution: the chromophore of green fluorescent protein," Faraday Discuss. 127, 149-63 (2004)

${ }^{22}$ Y. Liu, C. Li, Z. Ren, S. Yan, and M. R. Bryce, "All-organic thermally activated delayed fluorescence materials for organic light-emitting diodes," Nat. Rev. Mater. 3, 1-20 (2018).

${ }^{23}$ G. W. Robinson and R. P. Frosch, "Theory of electronic energy relaxation in the solid phase,"'J. Chem. Phys. 37, 1962-1973 (1962) 
${ }^{24} \mathrm{G}$. W. Robinson and R. P. Frosch, "Electronic excitation transfer and relaxation," J. Chem. Phys. 38, 1187-1203 (1963)

${ }^{25} \mathrm{~S}$. H. Lin, "Rate of interconversion of electronic and vibrational energy," J. Chem. Phys. 44, 3759-3767 (1966)

${ }^{26} \mathrm{~S}$. H. Lin and R. Bersohn, "Effect of partial deuteration and temperature on triplet-state lifetimes," J. Chem. Phys. 48, 2732-2736 (1968)

${ }^{27}$ Y. Niu, Q. Peng, and Z. Shuai, "Promoting-mode free formalism for excited state radiationless decay process with duschinsky rotation effect," Science in China Series B: Chemistry 51, 1153-1158 (2008).

${ }^{28}$ Y. Niu, Q. Peng, C. Deng, X. Gao, and Z. Shuai, "Theory of excited state decays and optical spectra: application to polyatomic molecules," J. Phys. Chem. A 114, 7817-7831 (2010).

${ }^{29}$ Q. Peng, Y. Niu, Q. Shi, X. Gao, and Z. Shuai, "Correlation function formalism for triplet excited state decay: Combined spin-orbit and nonadiabatic couplings," J. Chem. Theory Comput. 9, 1132-43 (2013)

${ }^{30} \mathrm{Q}$. Peng, Y. Yi, Z. Shuai, and J. Shao, "Toward quantitative prediction of molecular fluorescence quantum efficiency: role of duschinsky rotation,' J. Am. Chem. Soc. 129, 9333-9 (2007)

${ }^{31}$ R. Ianconescu and E. Pollak, "Semiclassical initial value representation study of internal conversion rates," J. Chem. Phys. 134, 234305 (2011)

${ }^{32}$ A. Humeniuk, M. Buzancic, J. Hoche, J. Cerezo, R. Mitric, F. Santoro, and V. Bonacic-Koutecky, "Predicting fluorescence quantum yields for molecules in solution: A critical assessment of the harmonic approximation and the choice of the lineshape function,"J. Chem. Phys. 152, 054107 (2020)

${ }^{33} \mathrm{~S}$. R. White, "Density matrix formulation for quantum renormalization groups,"'Phys. Rev. Lett. 69, 2863-2866 (1992)

${ }^{34}$ S. R. White, "Density-matrix algorithms for quantum renormalization groups," Phys. Rev. B 48, 10345-10356 (1993)

${ }^{35} \mathrm{G}$. Vidal, "Efficient simulation of one-dimensional quantum many-body systems," Phys. Rev. Lett. 93, 040502 (2004).

${ }^{36} \mathrm{~S}$. R. White and A. E. Feiguin, "Real-time evolution using the density matrix renormalization group," Phys. Rev. Lett. 93, 076401 (2004).

${ }^{37}$ A. W. Chin, J. Prior, R. Rosenbach, F. Caycedo-Soler, S. F. Huelga, and M. B. Plenio, "The role of non-equilibrium vibrational structures in electronic coherence and recoherence in pigment-protein complexes," Nat. Phys. 9, 113-118 (2013)

${ }^{38}$ E. Ronca, Z. Li, C. A. Jimenez-Hoyos, and G. K.-L. Chan, “Time-step targeting time-dependent and dynamical density matrix renormalization group algorithms with ab initio hamiltonians," J. Chem. Theory Comput. 13, 5560-5571 (2017).

${ }^{39}$ Y. Yao, K.-W. Sun, Z. Luo, and H. Ma, "Full quantum dynamics simulation of a realistic molecular system using the adaptive time-dependent density matrix renormalization group method," J. Phys. Chem. Lett. 9, 413-419 (2018).

${ }^{40}$ J. Ren, Z. Shuai, and G. Kin-Lic Chan, "Time-dependent density matrix renormalization group algorithms for nearly exact absorption and fluorescence spectra of molecular aggregates at both zero and finite temperature," J. Chem. Theory Comput. 14, 5027-5039 (2018)

${ }^{41}$ A. Baiardi and M. Reiher, "Large-scale quantum dynamics with matrix product states," J. Chem. Theory Comput. 15, 3481-3498 (2019).

${ }^{42}$ B. Kloss, D. R. Reichman, and R. Tempelaar, "Multiset matrix product state calculations reveal mobile franck-condon excitations under strong holstein-type coupling,"'Phys. Rev. Lett. 123, 126601 (2019)

${ }^{43}$ X. Xie, Y. Liu, Y. Yao, U. Schollwock, C. Liu, and H. Ma, "Timedependent density matrix renormalization group quantum dynamics for realistic chemical systems," J. Chem. Phys. 151, 224101 (2019)

${ }^{44} \mathrm{~W}$. Li, J. Ren, and Z. Shuai, "Finite-temperature td-dmrg for the carrier mobility of organic semiconductors," J. Phys. Chem. Lett. 11, 4930-4936 (2020).

${ }^{45}$ C. Hubig, I. McCulloch, and U. Schollwöck, "Generic construction of efficient matrix product operators," Phys. Rev. B 95, 035129 (2017).

${ }^{46}$ J. Ren, W. Li, T. Jiang, and Z. Shuai, "A general automatic method for optimal construction of matrix product operators using bipartite graph theory," J. Chem. Phys. 153, 084118 (2020).

${ }^{47}$ A. Jäckle and H.-D. Meyer, "Product representation of potential energy surfaces," J. Chem. Phys. 104, 7974-7984 (1996).

${ }^{48} \mathrm{~S}$. Manzhos and T. Carrington Jr, "Using neural networks to represent potential surfaces as sums of products," J. Chem. Phys. 125, 194105 (2006).
${ }^{49}$ A. E. Feiguin and S. R. White, "Finite-temperature density matrix renormalization using an enlarged hilbert space," Phys. Rev. B 72, 220401 (2005)

${ }^{50} \mathrm{U}$. Schollwöck, "The density-matrix renormalization group in the age of matrix product states," Ann. Phys. 326, 96-192 (2011).

${ }^{51}$ V. Barone, M. Biczysko, J. Bloino, M. Borkowska-Panek, I. Carnimeo, and P. Panek, "Toward anharmonic computations of vibrational spectra for large molecular systems," Int. J. Quantum Chem. 112, 2185-2200 (2012).

${ }^{52} \mathrm{M}$. Sibaev and D. Crittenden, "An efficient and numerically stable procedure for generating sextic force fields in normal mode coordinates," J. Chem. Phys. 144, 214107 (2016).

${ }^{53} \mathrm{G}$. Li, C. Rosenthal, and H. Rabitz, "High dimensional model representations," J. Phys. Chem. A 105, 7765-7777 (2001).

${ }^{54}$ J. M. Bowman, S. Carter, and X. Huang, "Multimode: a code to calculate rovibrational energies of polyatomic molecules," Int. Rev. Phys. Chem. 22, 533-549 (2003).

${ }^{55}$ O. Vendrell, F. Gatti, D. Lauvergnat, and H.-D. Meyer, "Full-dimensional (15-dimensional) quantum-dynamical simulation of the protonated water dimer. i. hamiltonian setup and analysis of the ground vibrational state," J. Chem. Phys. 127, 184302 (2007).

${ }^{56} \mathrm{~J}$. R. Reimers, "A practical method for the use of curvilinear coordinates in calculations of normal-mode-projected displacements and duschinsky rotation matrices for large molecules," J. Chem. Phys. 115, 9103-9109 (2001).

${ }^{57} \mathrm{~A}$. Baiardi, J. Bloino, and V. Barone, "Accurate simulation of resonanceraman spectra of flexible molecules: An internal coordinates approach," J. Chem. Theory Comput. 11, 3267-3280 (2015).

${ }^{58}$ J. Light, I. Hamilton, and J. Lill, "Generalized discrete variable approximation in quantum mechanics," J. Chem. Phys. 82, 1400-1409 (1985).

${ }^{59}$ S. Paeckel, T. Köhler, A. Swoboda, S. R. Manmana, U. Schollwöck, and C. Hubig, "Time-evolution methods for matrix-product states," Ann. Phys. 411, 167998 (2019).

${ }^{60} \mathrm{~W}$. Li, J. Ren, and Z. Shuai, "Numerical assessment for accuracy and gpu acceleration of td-dmrg time evolution schemes," J. Chem. Phys. 152, 024127 (2020)

${ }^{61}$ H.-D. Meyer and H. Wang, "On regularizing the mctdh equations of motion," J. Chem. Phys. 148, 124105 (2018).

62"Renormalizer," https://github.com/shuaigroup/Renormalizer

${ }^{63}$ X.-K. Chen, Y. Tsuchiya, Y. Ishikawa, C. Zhong, C. Adachi, and J.-L. Brédas, "A new design strategy for efficient thermally activated delayed fluorescence organic emitters: from twisted to planar structures," Adv. Mater. 29, 1702767 (2017).

${ }^{64}$ M. J. Frisch, G. W. Trucks, H. B. Schlegel, G. E. Scuseria, M. A. Robb, J. R. Cheeseman, G. Scalmani, V. Barone, G. A. Petersson, H. Nakatsuji, X. Li, M. Caricato, A. V. Marenich, J. Bloino, B. G. Janesko, R. Gomperts, B. Mennucci, H. P. Hratchian, J. V. Ortiz, A. F. Izmaylov, J. L. Sonnenberg, D. Williams-Young, F. Ding, F. Lipparini, F. Egidi, J. Goings, B. Peng, A. Petrone, T. Henderson, D. Ranasinghe, V. G. Zakrzewski, J. Gao, N. Rega, G. Zheng, W. Liang, M. Hada, M. Ehara, K. Toyota, R. Fukuda, J. Hasegawa, M. Ishida, T. Nakajima, Y. Honda, O. Kitao, H. Nakai, T. Vreven, K. Throssell, J. A. Montgomery, Jr., J. E. Peralta, F. Ogliaro, M. J. Bearpark, J. J. Heyd, E. N. Brothers, K. N. Kudin, V. N. Staroverov, T. A. Keith, R. Kobayashi, J. Normand, K. Raghavachari, A. P. Rendell, J. C. Burant, S. S. Iyengar, J. Tomasi, M. Cossi, J. M. Millam, M. Klene, C. Adamo, R. Cammi, J. W. Ochterski, R. L. Martin, K. Morokuma, O. Farkas, J. B. Foresman, and D. J. Fox, "Gaussian 16 Revision C.01," (2016), gaussian Inc. Wallingford CT.

${ }^{65} \mathrm{Z}$. Shuai, "Thermal vibration correlation function formalism for molecular excited state decay rates," Chin. J. Chem 38, 1223-1232 (2020).

${ }^{66}$ O. Christiansen, "Midascpp," https://gitlab.com/midascpp/ midascpp

${ }^{6 /}$ M. Sparta, D. Toffoli, and O. Christiansen, "An adaptive density-guided approach for the generation of potential energy surfaces of polyatomic molecules," Theor. Chem. Acc. 123, 413-429 (2009).

${ }^{68}$ A. Nitzan, Chemical dynamics in condensed phases: relaxation, transfer and reactions in condensed molecular systems (Oxford university press, 2006).

${ }^{69}$ J. Mei, N. L. Leung, R. T. Kwok, J. W. Lam, and B. Z. Tang, "Aggregationinduced emission: together we shine, united we soar!" Chem. Rev. 115, 11718-11940 (2015). 


\title{
Supplementary materials for "Evaluating the anharmonicity contributions to the molecular excited state internal conversion rates with finite temperature TD-DMRG"
}

\author{
Yuanheng Wang, Jiajun Ren.** and Zhigang Shuai \\ MOE Key Laboratory of Organic OptoElectronics and Molecular Engineering, \\ Department of Chemistry, Tsinghua University, Beijing 100084, People's Republic of China \\ (Dated: May 28, 2021)
}

\section{DISCRETE VARIABLE REPRESENTATION}

Discrete variable representation (DVR) basis is a basis set composed of series of local distributed basis functions in the $\delta$ function form on the position coordinate. It can be obtained through diagonalization of the matrix representation of position operator $\hat{x}$ on another basis set as in Eq. 2. Here, we use the harmonic oscillator (HO) basis as an example, the relationship between the primitive basis and DVR basis transformed can be expressed as Eq. 3 and Eq. 4 .

$$
\begin{gathered}
X^{\mathrm{HO}}=\sum_{\alpha \beta}\left\langle\phi_{\alpha}^{\mathrm{HO}}|\hat{x}| \phi_{\beta}^{\mathrm{HO}}\right\rangle\left|\phi_{\alpha}^{\mathrm{HO}}\right\rangle\left\langle\phi_{\beta}^{\mathrm{HO}}\left|=\sum_{\alpha \beta} X_{\alpha \beta}^{\mathrm{HO}}\right| \phi_{\alpha}^{\mathrm{HO}}\right\rangle\left\langle\phi_{\beta}^{\mathrm{HO}}\right| \\
X^{\mathrm{DVR}}=U^{\dagger} X^{\mathrm{HO}} U \\
\left|\phi_{\beta}^{\mathrm{DVR}}\right\rangle=\sum_{\beta^{\prime}}\left|\phi_{\beta^{\prime}}^{\mathrm{HO}}\right\rangle U_{\beta^{\prime} \beta} \\
\left|\phi_{\beta}^{\mathrm{HO}}\right\rangle=\sum_{\beta^{\prime}}\left|\phi_{\beta^{\prime}}^{\mathrm{DVR}}\right\rangle U_{\beta^{\prime} \beta}^{\dagger}
\end{gathered}
$$

Using the local distribution feature of the basis functions in the DVR basis on position coordinate, the matrix elements $V^{\mathrm{DVR}}$ of operators $\hat{V}(x)$ with an explicit relationship with the position operator $\hat{x}$ on DVR basis can be easily written through Eq. 5

$$
V_{\alpha \beta}^{\mathrm{DVR}}=\left\langle\phi_{\alpha}^{\mathrm{DVR}}|\hat{V}| \phi_{\beta}^{\mathrm{DVR}}\right\rangle \approx V\left(x_{\alpha}\right) \delta_{\alpha \beta}
$$

Then, a linear transformation as in Eq. 6 and Eq.7 can be performed if we need the matrix representation $V^{\mathrm{HO}}$ of the operator $\hat{V}$ in the original $\mathrm{HO}$ basis.

$$
\begin{gathered}
V_{\alpha \beta}^{\mathrm{HO}}=\left\langle\phi_{\alpha}^{\mathrm{HO}}|\hat{V}| \phi_{\beta}^{\mathrm{HO}}\right\rangle=\sum_{\alpha^{\prime} \beta^{\prime}} U_{\alpha \alpha^{\prime}}\left\langle\phi_{\alpha^{\prime}}^{\mathrm{DVR}}|\hat{V}| \phi_{\beta^{\prime}}^{\mathrm{DVR}}\right\rangle U_{\beta^{\prime} \beta}^{\dagger} \\
V^{\mathrm{HO}}=U V^{\mathrm{DVR}} U^{\dagger}
\end{gathered}
$$

In this work, it is hard to analytically write the matrix elements of Morse potential operator $\hat{V}^{\text {Morse }}=D\left(1-e^{-\alpha \hat{x}}\right)^{2}$ on the HO basis set. So we first write its matrix elements on HO-DVR basis as Eq. 5. Then we transform the obtained matrix representation $V^{\mathrm{DVR}}$ to the matrix representation $V^{\mathrm{HO}}$ in the original chosen $\mathrm{HO}$ basis through a transformation matrix $U$ between the two basis sets.

\section{THE CONVERGENCE OF VIRTUAL BOND DIMENSION AND PHYSICAL BOND DIMENSION OF TD-DMRG SIMULATION IN THE TWO-MODE MODEL SYSTEM}

renji@mail.tsinghua.edu.cn

zgshuai@tsinghua.edu.cn 

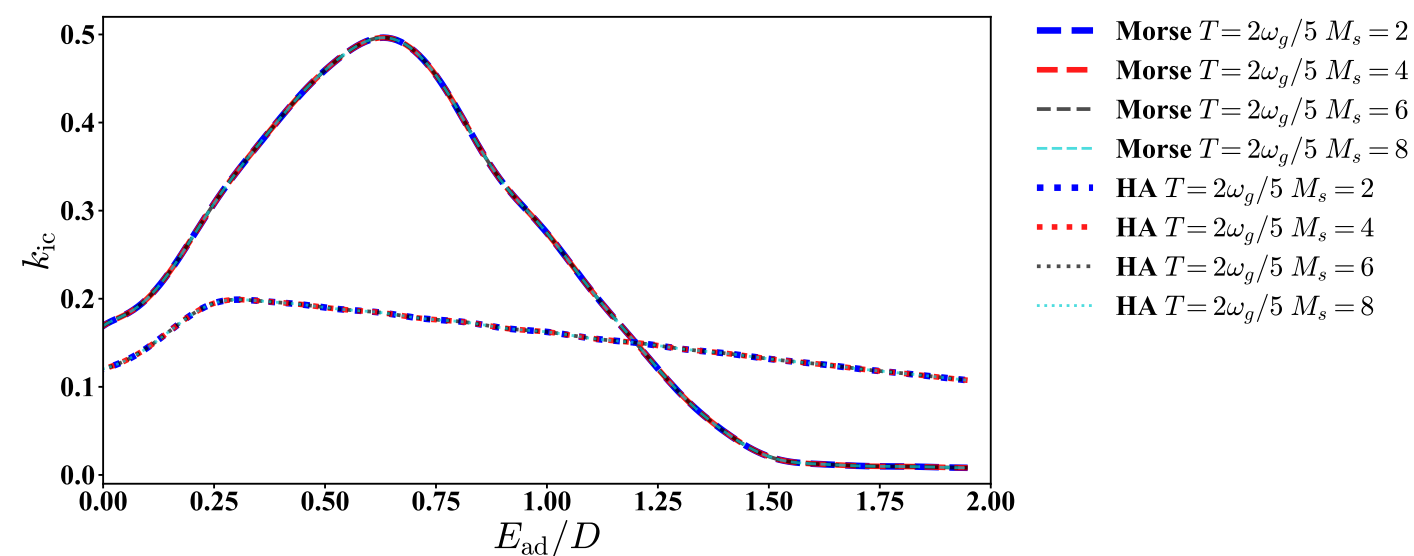

FIG. 1. The convergence of the bond dimensions $M_{S}$ for MPS in the two-mode model used in the main text. The displacement $\Delta q=0.7 / \alpha$. the temperature $T=2 \omega_{g} / 5$, the physical bond dimension $d=100$.The result indicates that $M_{S}=4$ is large enough in this calculation.
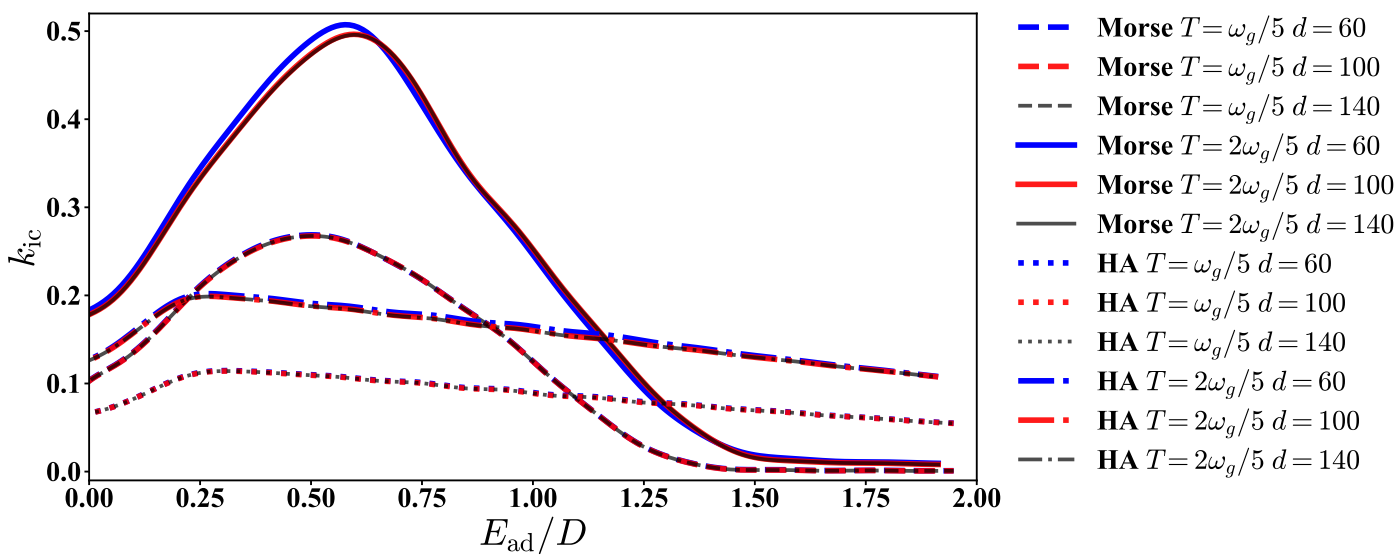

FIG. 2. The convergence of the primitive basis set (simple harmonic oscillator basis) in the two-mode model used in the main text. The displacement $\Delta q=0.7 / \alpha$. With all the temperatures considered, the results with physical bond dimension $d=100$ and $d=140$ are the same, indicating that $d=100$ is large enough in this calculation.
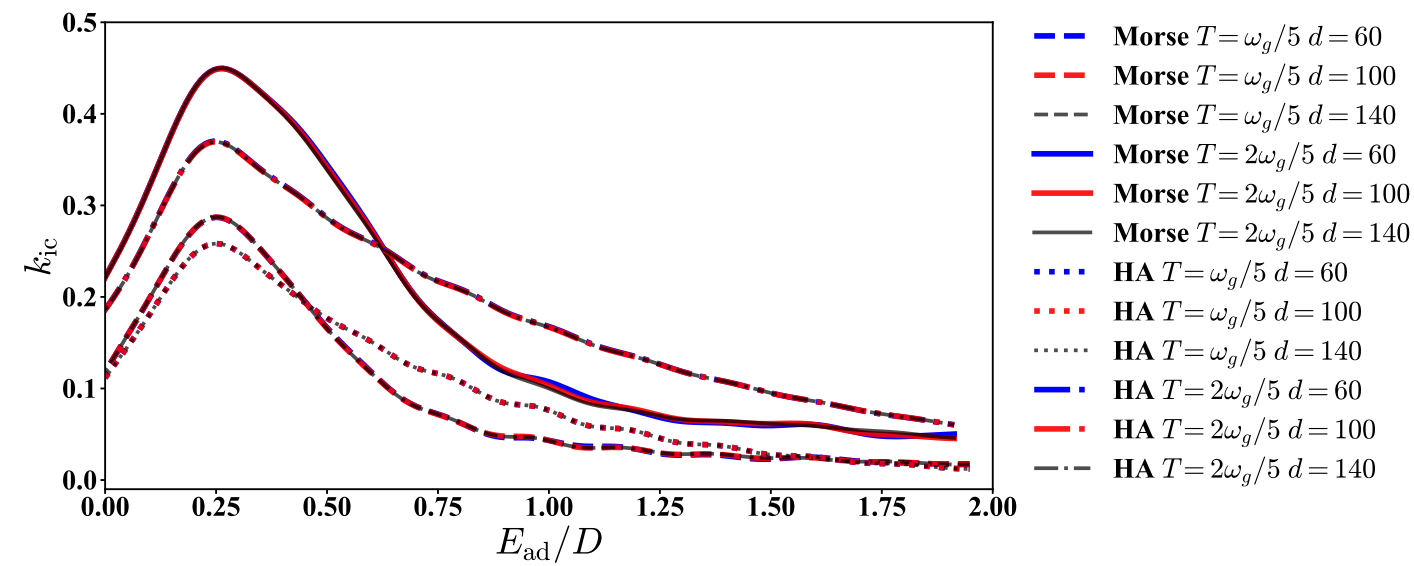

FIG. 3. The convergence of the primitive basis set (simple harmonic oscillator basis) in the two-mode model used in the main text. The displacement $\Delta q=0 / \alpha$. With all the temperatures considered, the results with physical bond dimension $d=100$ and $d=140$ are the same, indicating that $d=100$ is large enough in this calculation. 


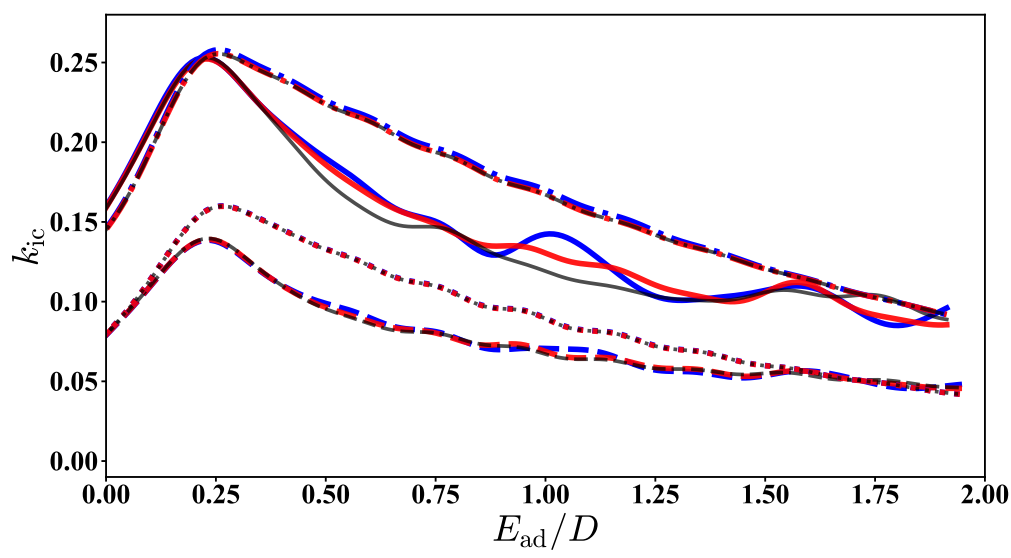

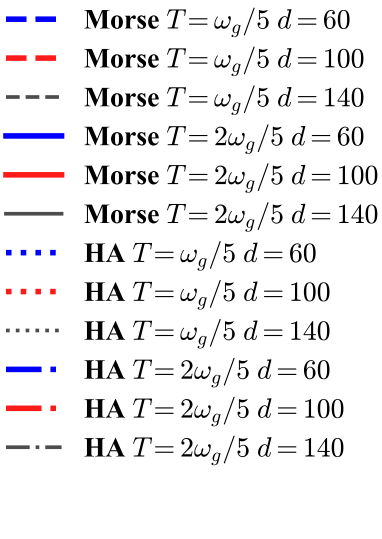

(a)

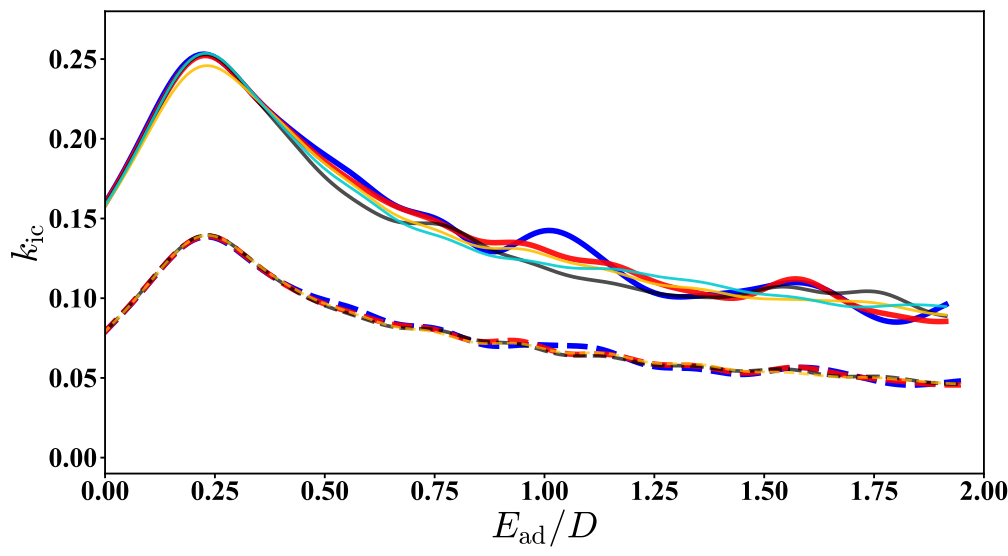

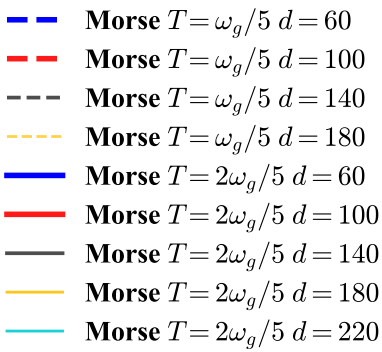

(b)

FIG. 4. (a). The convergence of the primitive basis set (simple harmonic oscillator basis) in the two-mode model used in the main text. The displacement $\Delta q=-0.52 / \alpha$. The results with $d=100$ are consistent with that of $d=140$ except the Morse potential with $T=2 \omega_{g} / 5$. (b). For Morse potential with $T=2 \omega_{g} / 5$, a larger basis set is tested. With $d$ up to $220, k_{\text {ic }}$ is still not converged in the high energy region. However, considering the error caused by the insufficient basis in this case does not change the relative relationship between the internal conversion rate calculated from Morse potential and its harmonic approximation, we still use $d=100$ in the main text for higher efficiency. 


\section{THE RESULTS OF THE TWO-MODE MODEL SYSTEM WITH DIFFERENT MORSE PARAMETERS}

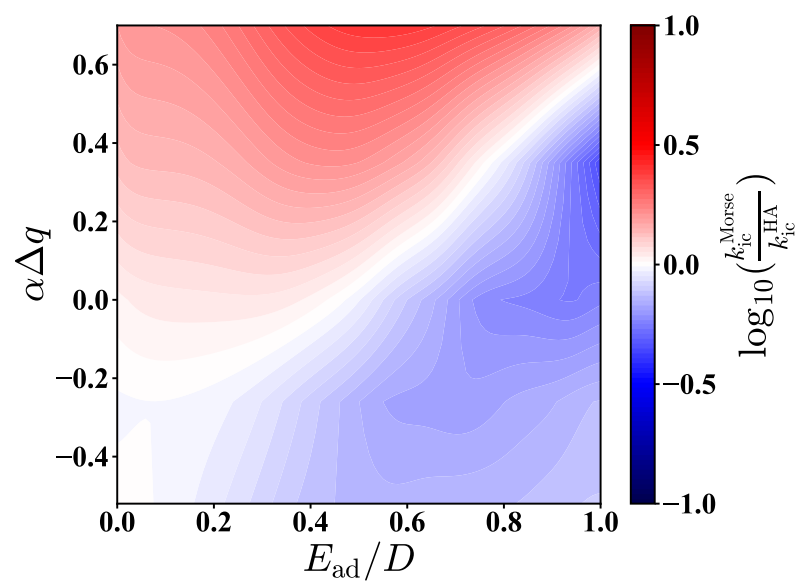

(a)

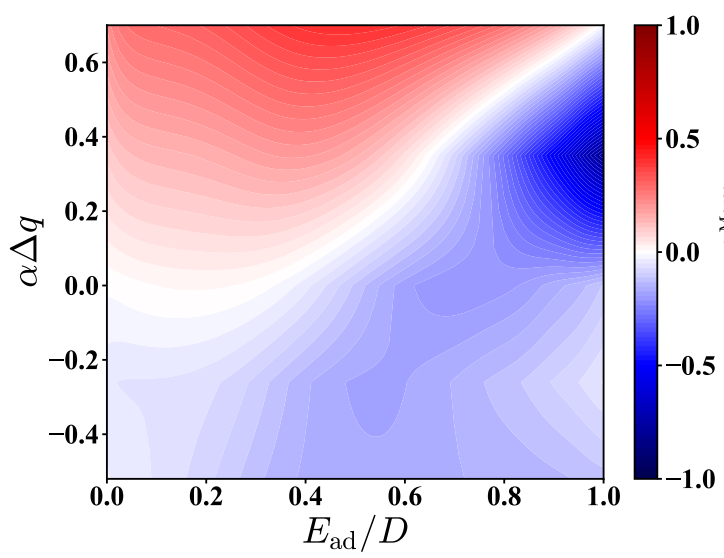

(b)

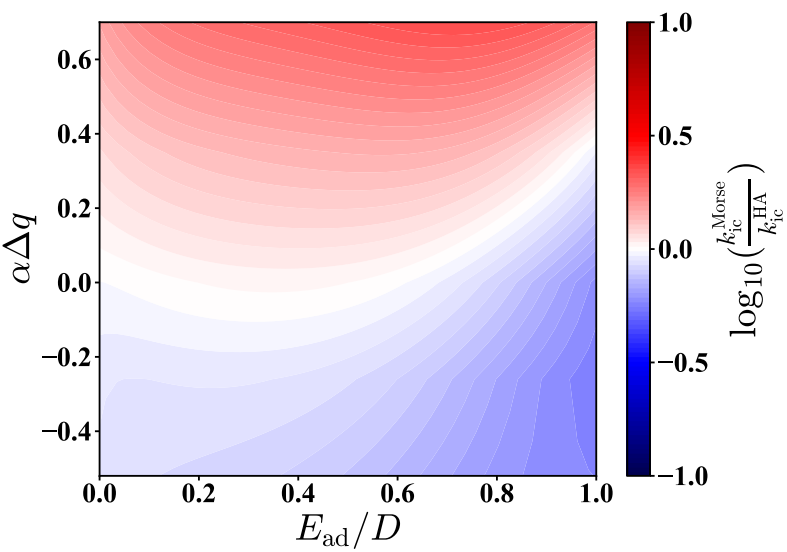

(c)

FIG. 5. (a), (b) and (c) show the ratio of the internal conversion rate on the Morse potential with respect to that under HA with different displacements and adiabatic excitation energies. (a). $D=D_{0}, \alpha=\alpha_{0}$. The same as Fig. 5a in the main text. (b). $D=D_{0}, \alpha=2 / 3 \alpha_{0}$. (c). $D=4 / 9 D_{0}, \alpha=\alpha_{0}$. In all cases, $D_{0}=5.52 \mathrm{eV}, \alpha_{0}=2.23 \mathrm{amu}^{-1 / 2} \AA^{-1}, \omega_{e}=\omega_{g} / 5$ and $T=\omega_{g} / 5$. The parameter for TD-DMRG calculations are all $d=100, M_{s}=4$.

\section{THE COMPUTATIONAL COMPLEXITY OF TD-DMRG IN THE MORSE MODEL SYSTEM WITH BI-MODE COUPLING AND WITH DIFFERENT SYSTEM SIZE}

Though we have focused on a two-independent-mode system in the main text, we expect the same method can be applied to larger systems, even with mode coupling. Thus, in this section, we evaluate the time cost of our methods for different systems sizes. Our benchmark platform is one CPU core on Intel Xeon® Gold 5115 CPU at 2.40 GHz with NVIDIA ${ }^{\circledR}$ Tesla ${ }^{\circledR}$ V100-PCIE-32 GB for CPU-GPU heterogeneous calculations.

In the first test case, only the number of modes increases from 2 to 20 . As we discussed in the main text, when the modes are uncoupled and additionally there is no mode-mixing between the excited state and ground state PESs, MPS with $M_{S}=2$ can always exactly represent the time-dependent wavefunction no matter what system size is. Since the formal time scaling of the time-evolution algorithm used in this work is $O\left(N\left(M_{S}^{2} M_{O}^{2} d^{2}+M_{S}^{3} M_{O} d+M_{S}^{3} d^{2}\right)\right)$, considering $M_{O}$ and $M_{S}$ are both unchanged, the time cost is linear with the system size. The numerical results are shown in FIG. \$6.

Since TD-DMRG can deal with correlated Hamiltonian, in the second test case, we apply the proposed method to a fullycoupled anharmonic potential. For simplicity, we add a fourth-order bi-mode coupling term to the potential (see Eq. 8). As shown in Fig. 7, the coupling strength $\gamma$ will influence the transition rate. In the following calculation, we set $\gamma=0.1$. Unlike the uncoupled case above, the bond dimension $M_{S}$ should be increased to achieve a convergent result when the system size increases. 
The numerical results with different $M_{s}$ for each system size are shown in Fig. 8 The time costs have been collected in FIG. 6b. Though the time cost is no more linear with the system size, it is still polynomial $\left(\sim N^{3}\right)$ as expected. The benchmark results demonstrate that our method has the potential to deal with larger systems with mode-couplings.

$$
\begin{gathered}
V_{f}=V_{\mathrm{g}}=\sum_{l} V_{l}^{\text {Morse }}+\Gamma(\gamma) \sum_{l<k} q_{\mathrm{g}, l}^{2} q_{\mathrm{g}, k}^{2} \\
\Gamma(\gamma)=\frac{\gamma}{4 !} \frac{\partial^{4} V^{\text {Morse }}}{\partial q_{\mathrm{g}, l}^{4}}\left(q_{\mathrm{g}, l}=0\right)=\frac{\gamma}{4 !} 14 \alpha^{4} D \\
V_{l}^{\text {Morse }}=D\left(1-e^{-\alpha q_{\mathrm{g}, l}}\right)^{2}
\end{gathered}
$$

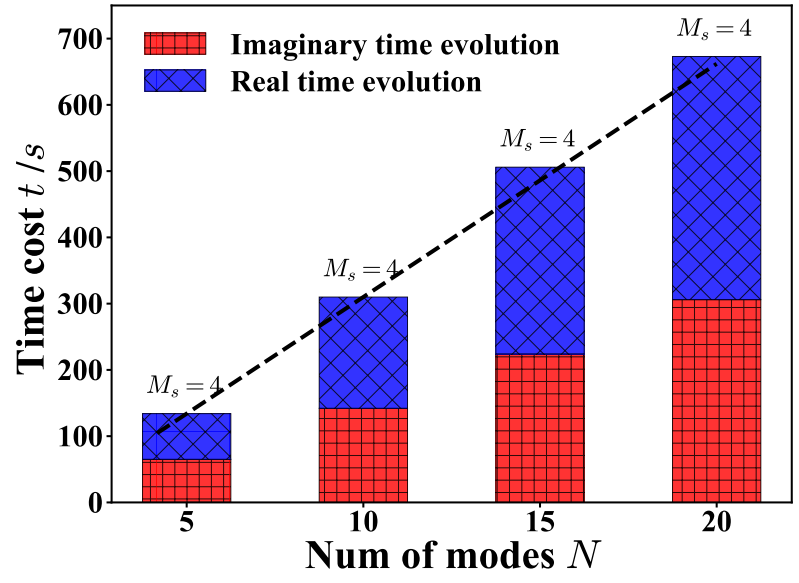

(a)

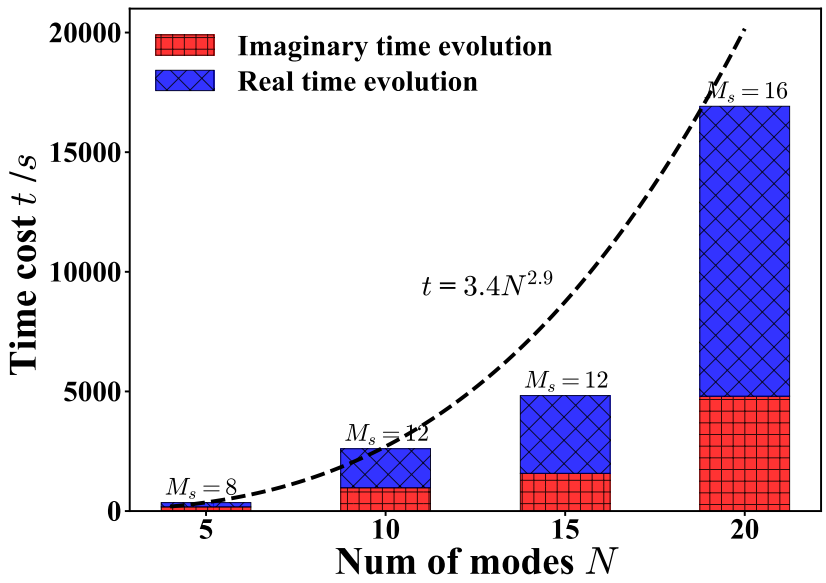

(b)

FIG. 6. The time cost of TD-DMRG with different system sizes using their corresponding minimum convergent virtual bond dimensions $M_{S}$ (list above the bars). The physical bond dimension is $d=100$ for all the calculations. (a) shows the results without mode-coupling (Eq. 8 with $\gamma=0$ ). (b) shows the results with mode-coupling (Eq. 8 with $\gamma=0.1$ ). The Morse potentials used along each mode all share the same parameters $\alpha, D$ and $\Delta q=0.7 / \alpha$ as in FIG. 5b in the main article, the temperature is $T=\omega_{\mathrm{g}} / 5$.

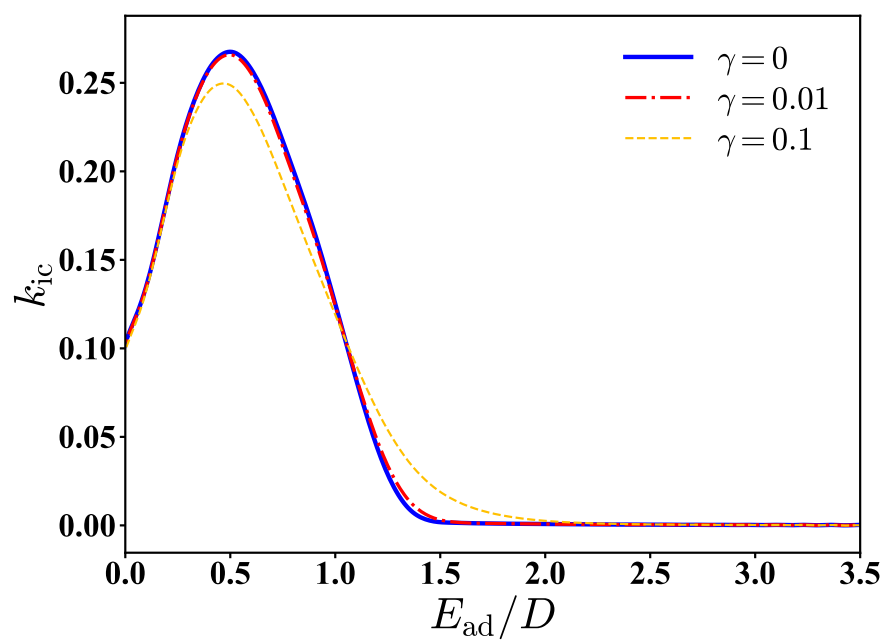

FIG. 7. The internal conversion rates calculated for different magnitude of mode-coupling strengths in the two-mode Morse model. The magnitude is controlled by a constant $\gamma$ introduced in Eq. 8). It can be seen $\gamma=0.1$ is enough to cause an obvious influence on the internal conversion rates. 


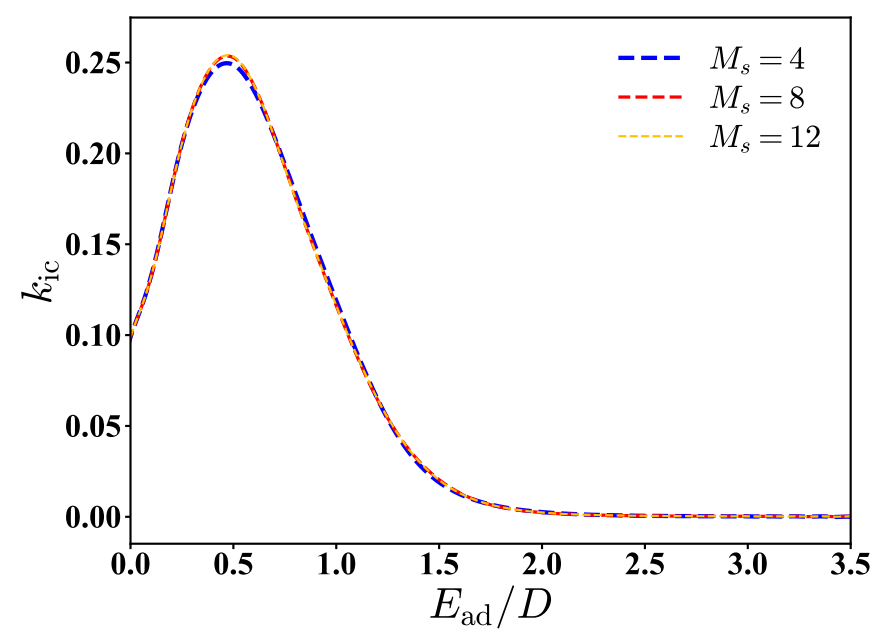

(a)

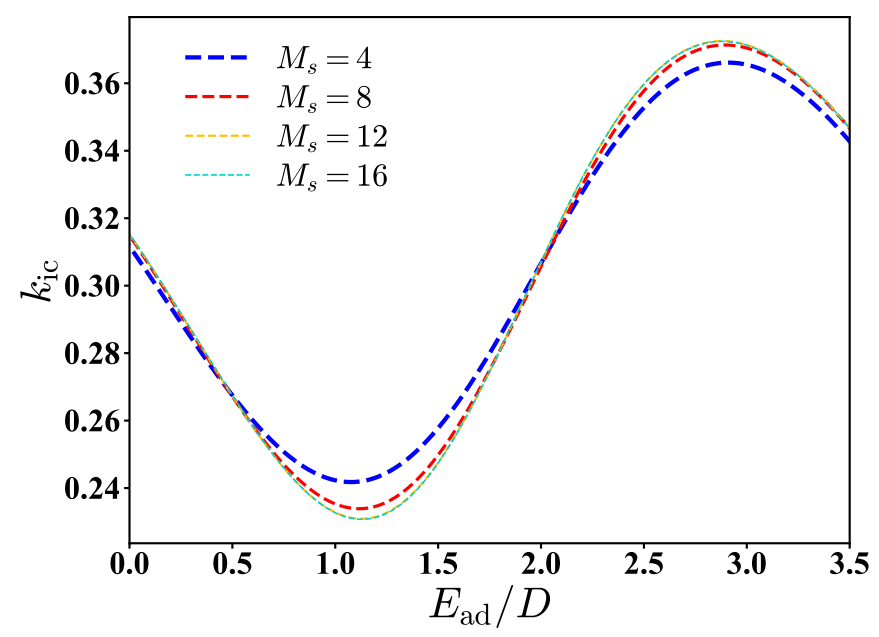

(c)

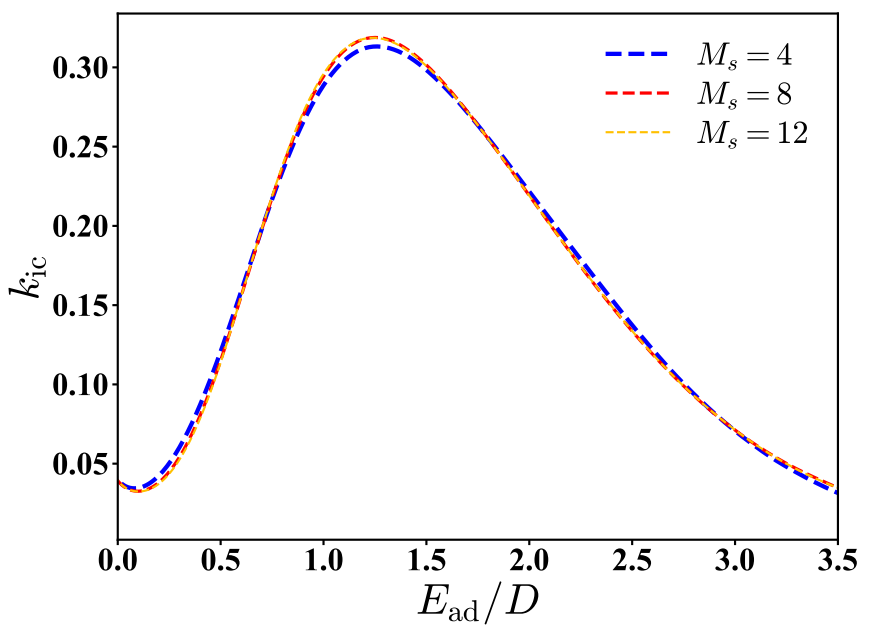

(b)

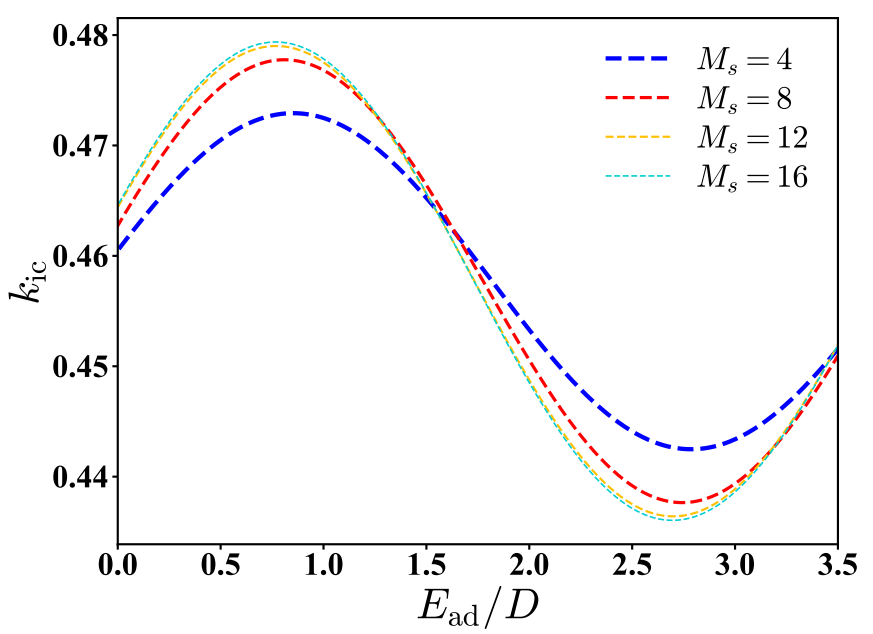

(d)

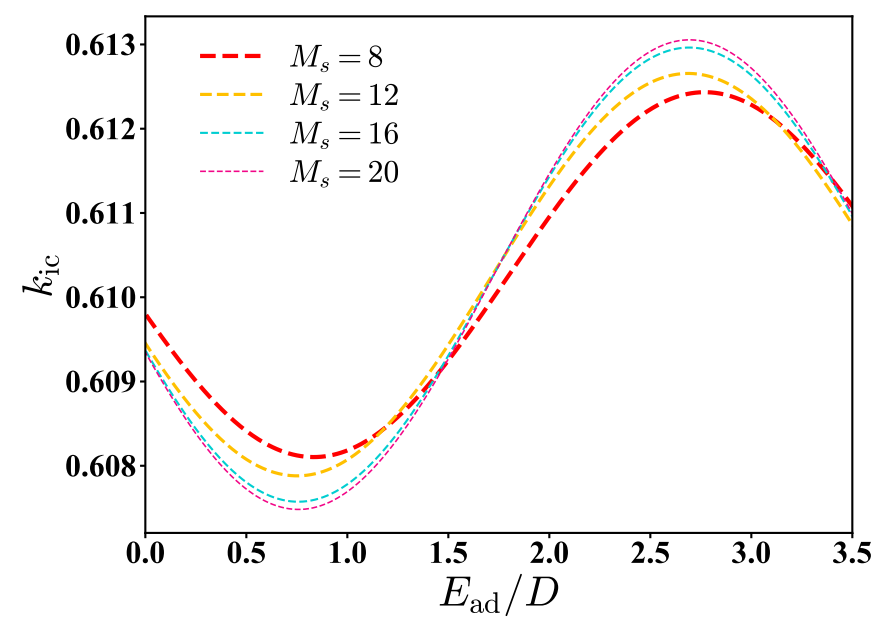

(e)

FIG. 8. (a), (b),(c),(d) and (e) show the convergence of the bond dimensions $M_{S}$ for MPS in the fully-coupled model with a different number of Morse modes (system size). The number of modes are (a) 2, (b) 5, (c) 10, (d) 15, and (e) 20 respectively. 


\section{THE 1-D CUT OF AZULENE GROUND STATE PES ALONG EACH NORMAL MODE}

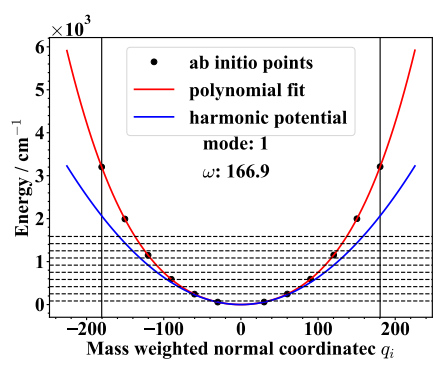

(a)

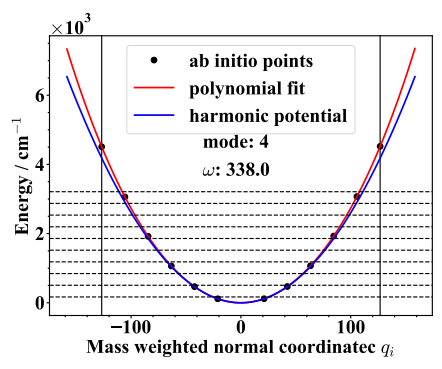

(d)

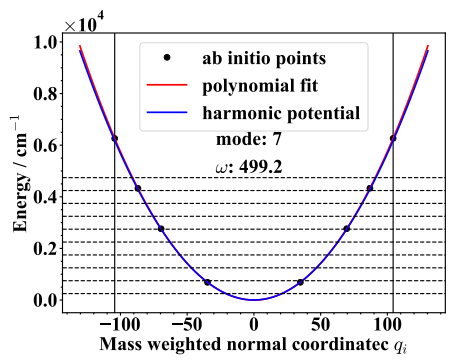

(g)

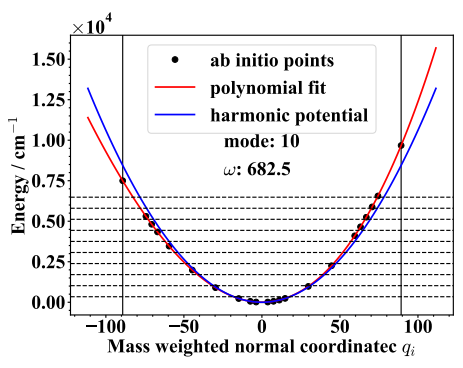

(j)

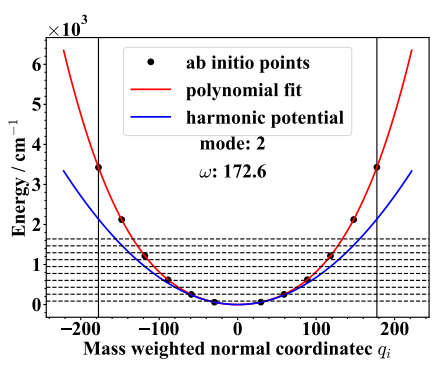

(b)

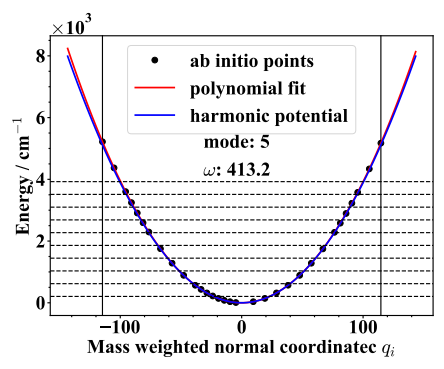

(e)

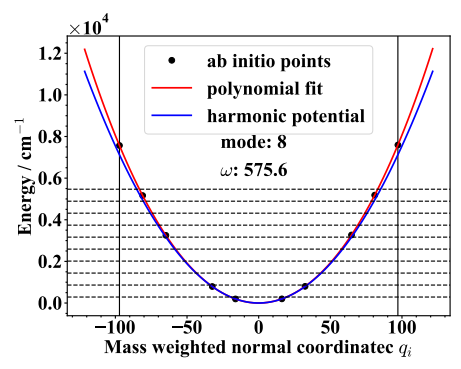

(h)

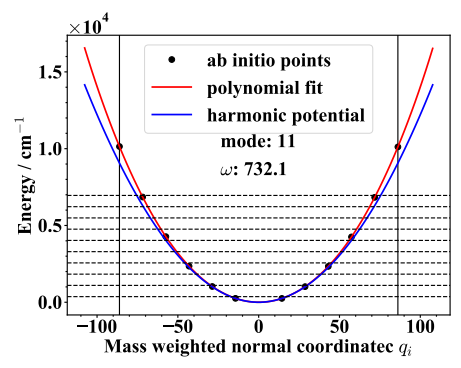

(k)

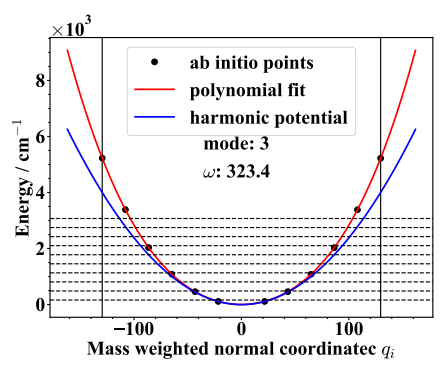

(c)

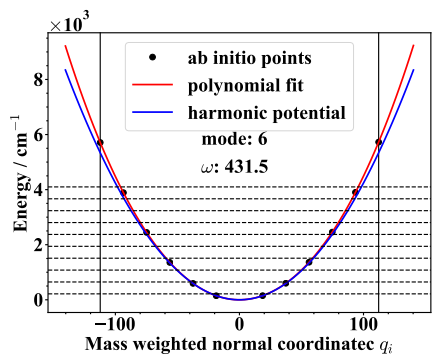

(f)

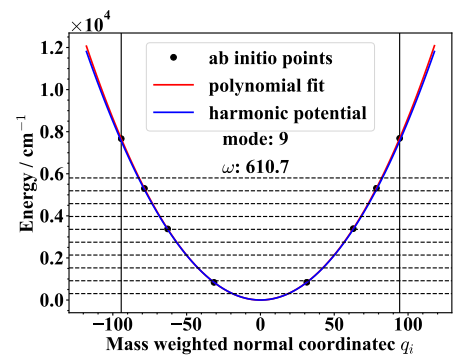

(i)

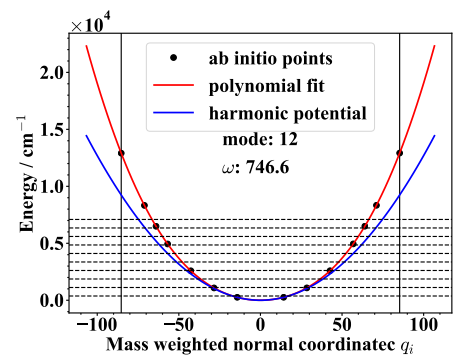

(1)

FIG. 9. (a)-(1) The 1-D ground state PES cut of azulene from mode 1 to mode 12. The unit of mass-weighted normal coordinates is the atomic unit. The solid circles are the ab initio points. The red line is the polynomial fit of ab initio points up to order 12 . The blue line is harmonic potential. The vertical solid line is the boundary of the ab initio points acting as a guide to the eye. The dashed horizon lines are the lowest 10 energy levels of the harmonic potential. The ab initio calculations are carried out at B3LYP/6-31g(d) level. 


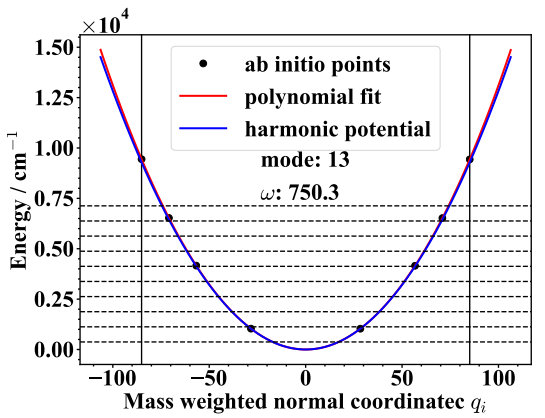

(a)

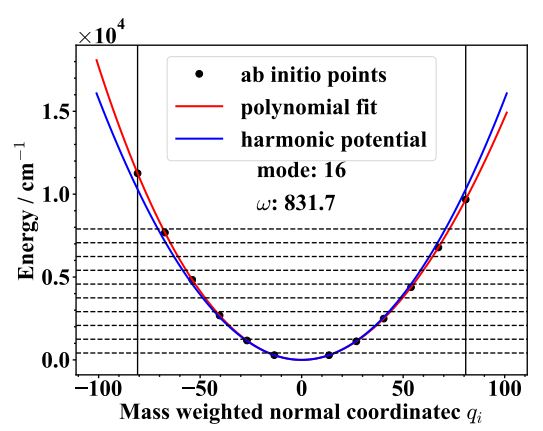

(d)

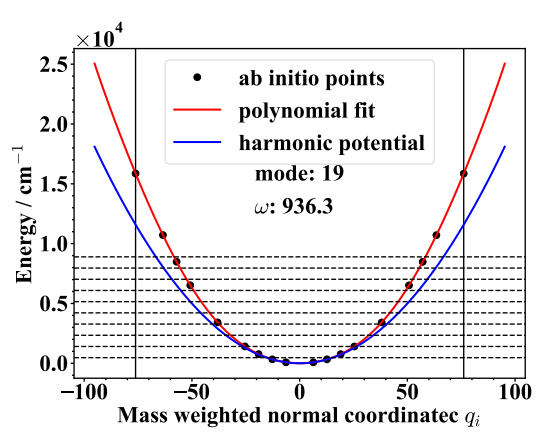

(g)

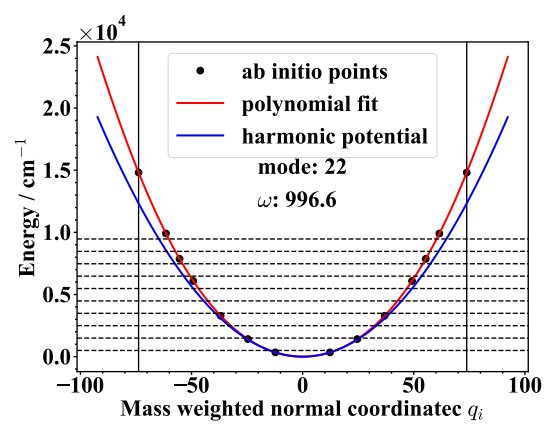

(j)

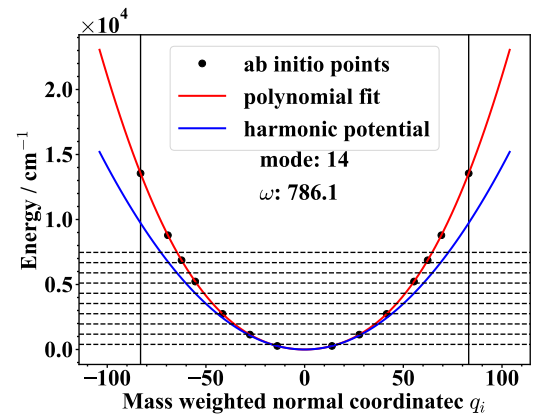

(b)

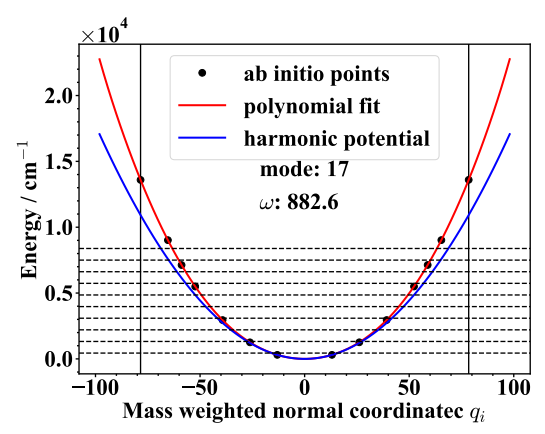

(e)

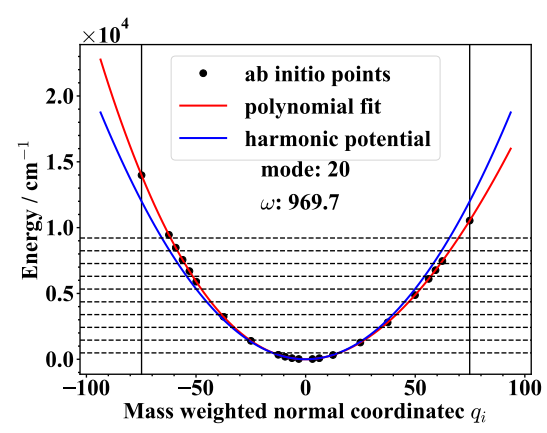

(h)

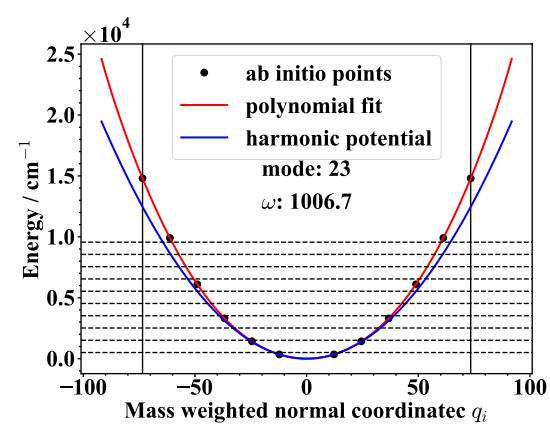

(k)

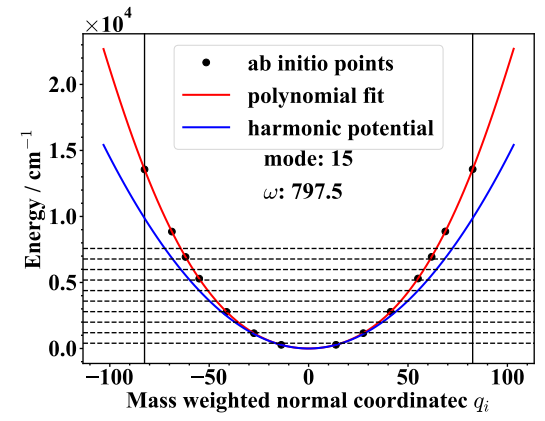

(c)

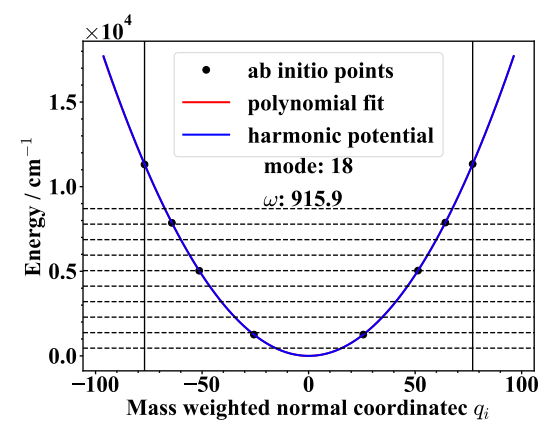

(f)

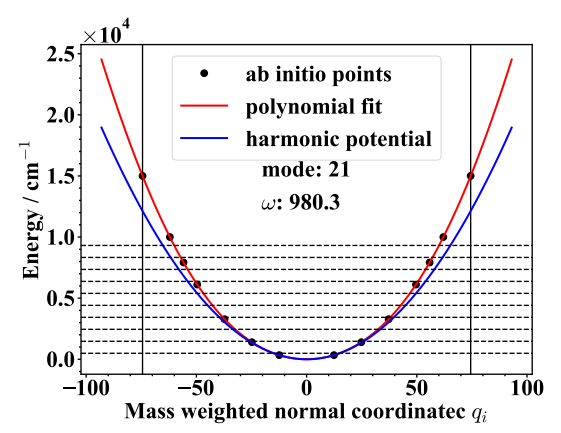

(i)

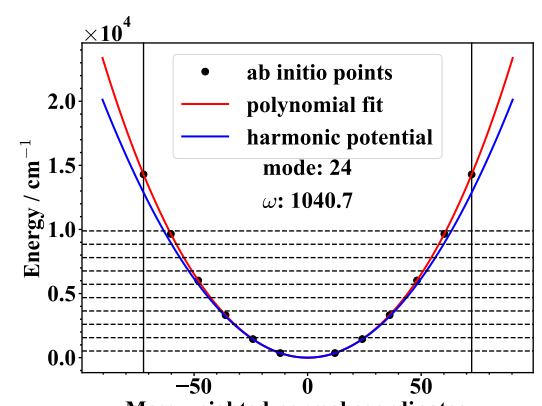

Mass weighted normal coordinatec $q_{i}$

(1)

FIG. 10. Similar as Fig. 9 but for modes 13-24. 


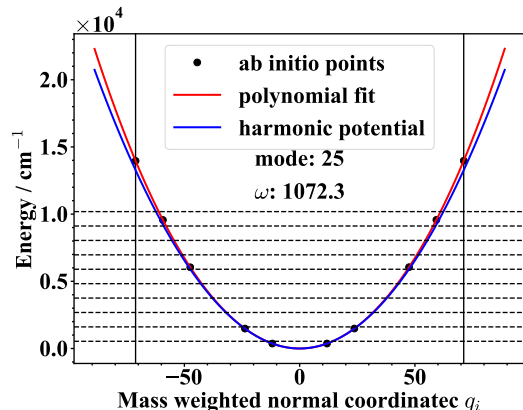

(a)

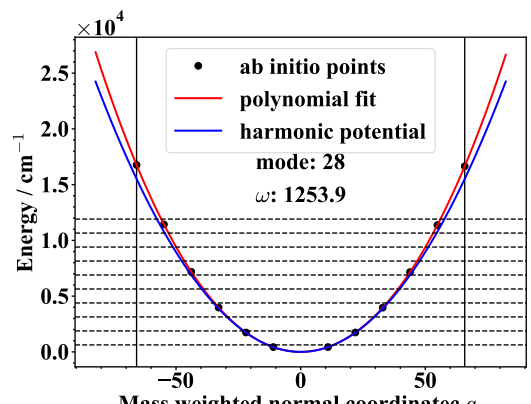

(d)

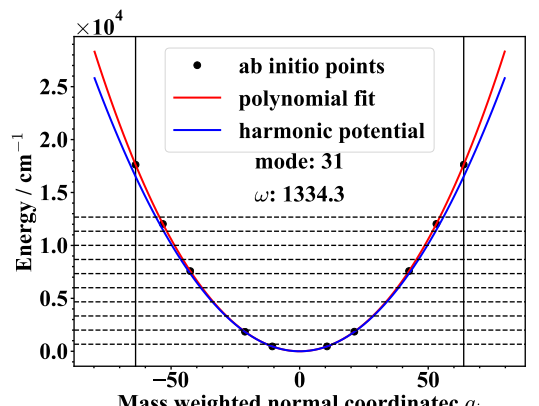

(g)

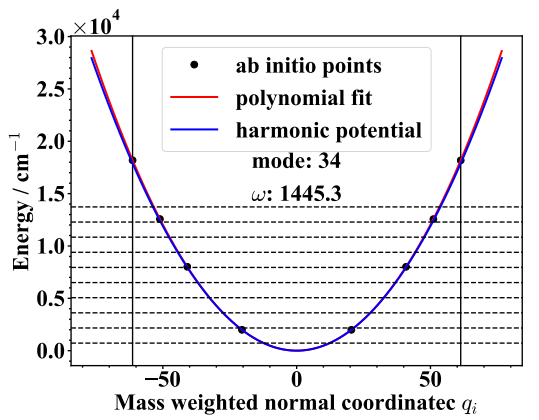

(j)

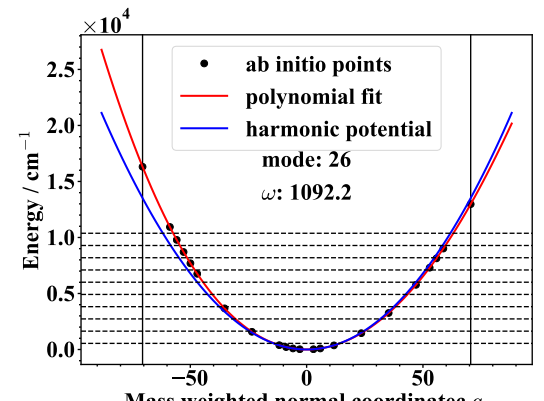

(b)

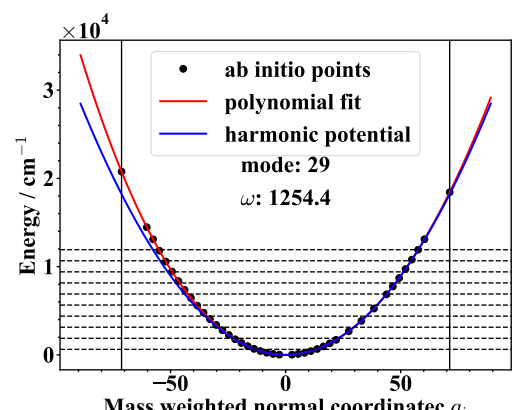

(e)

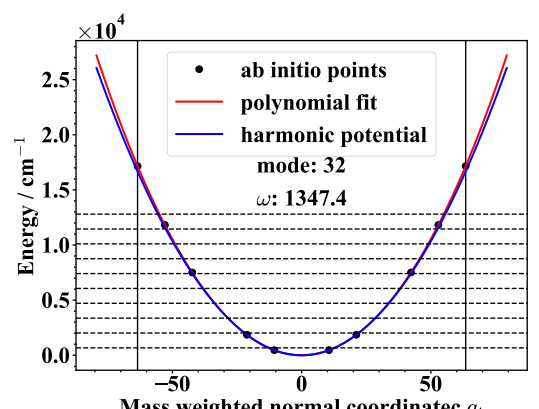

(h)

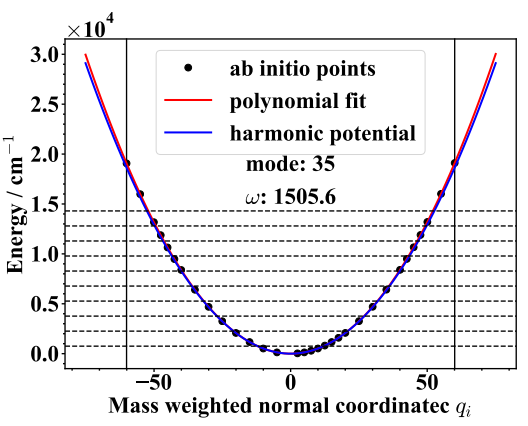

(k)

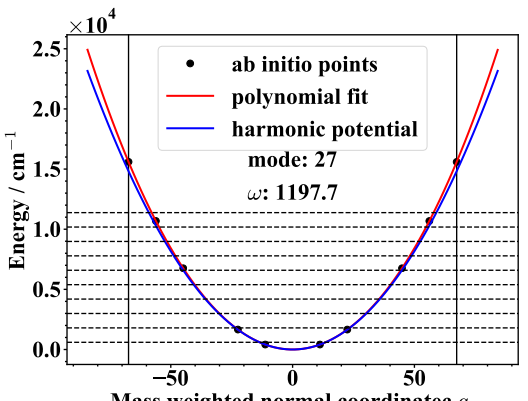

Mass weighted normal coordinatec $q_{i}$

(c)

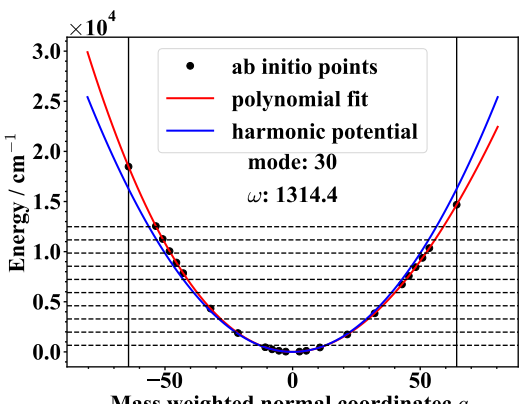

(f)

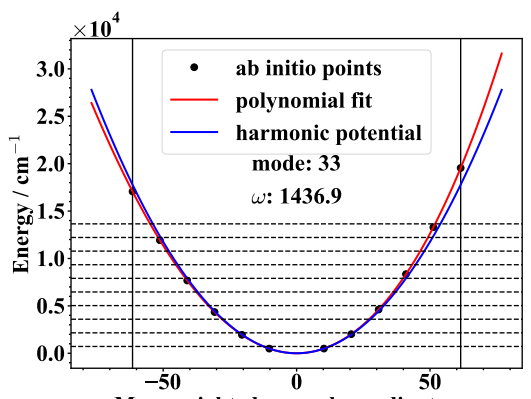

Mass weighted normal coordinatec $q_{i}$

(i)

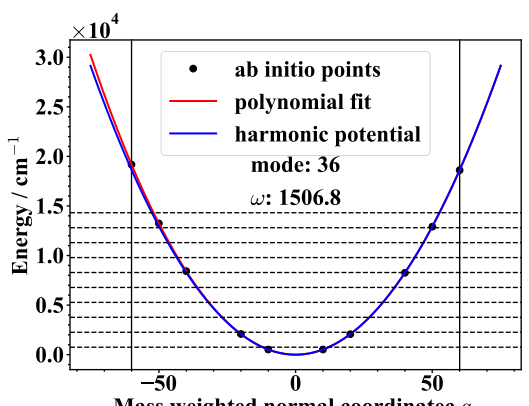

(1)

FIG. 11. Similar as Fig. 9 but for modes 25-36. 


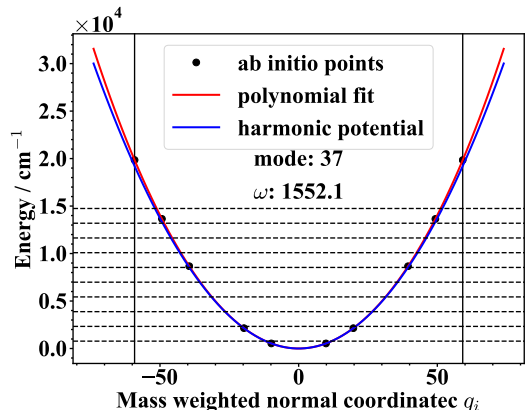

(a)

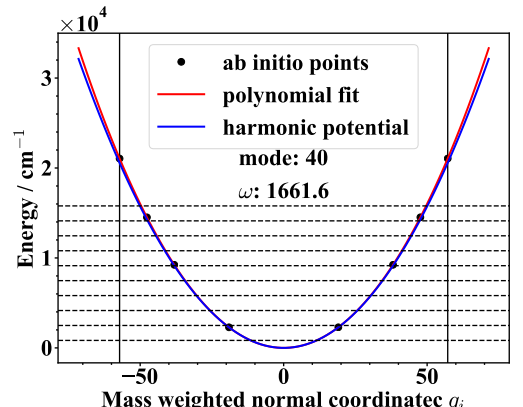

(d)

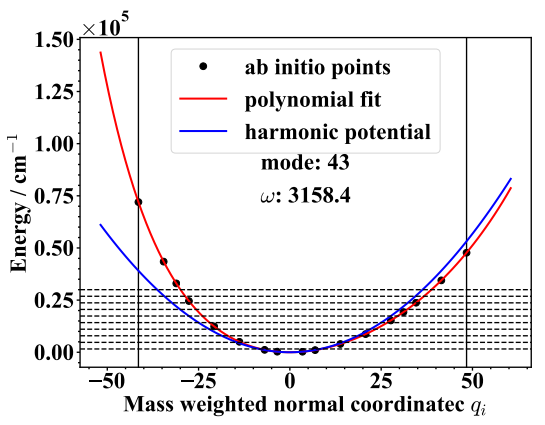

(g)

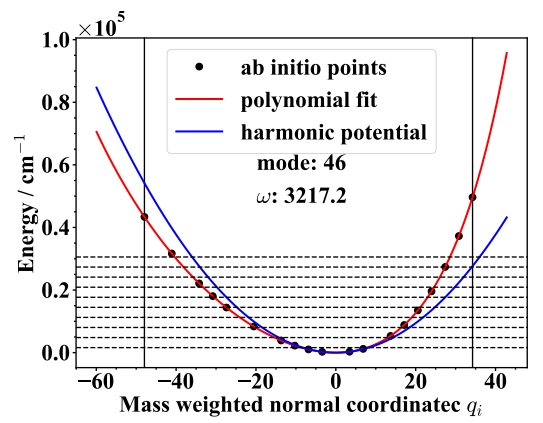

(j)

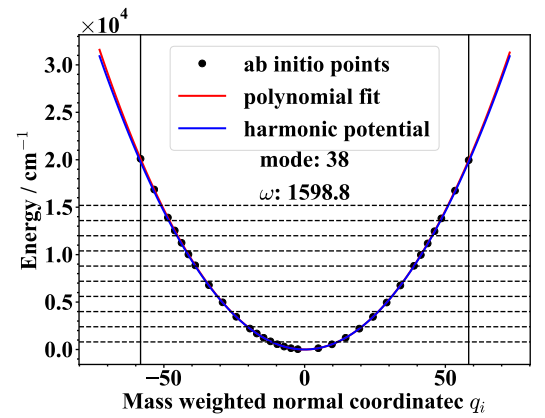

(b)

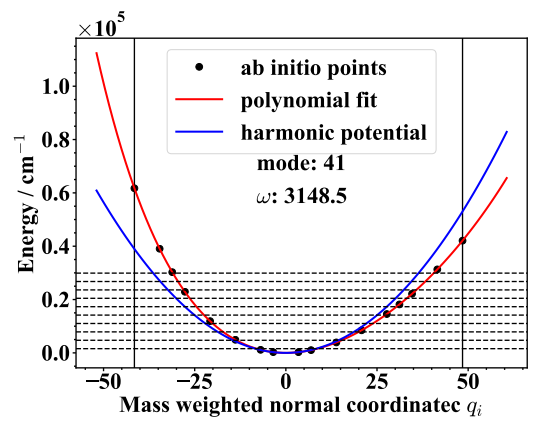

(e)

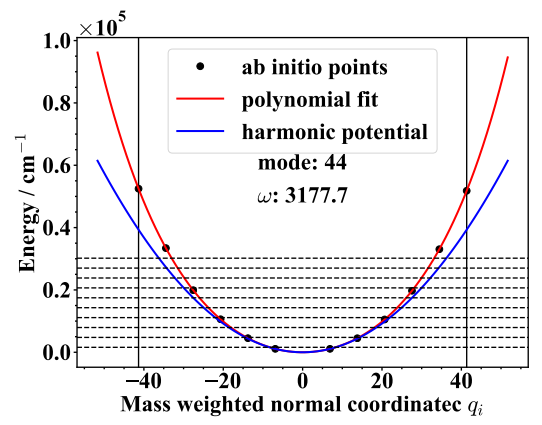

(h)

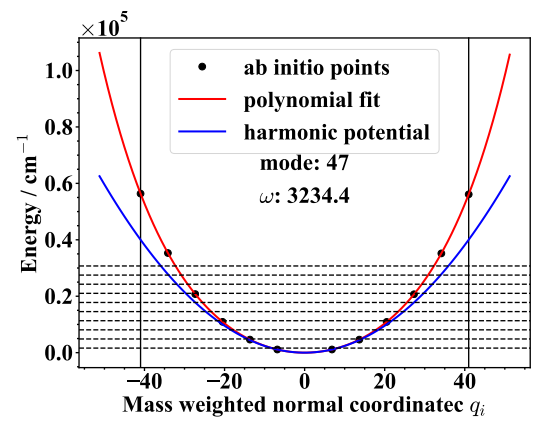

(k)

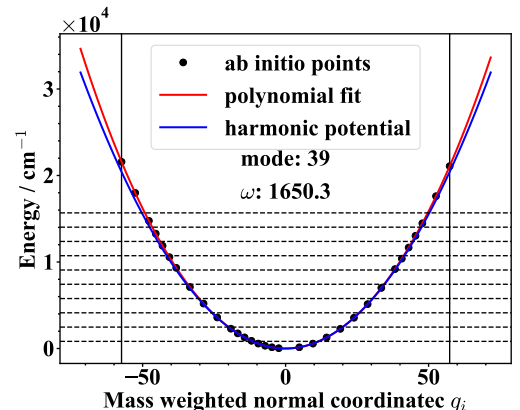

(c)

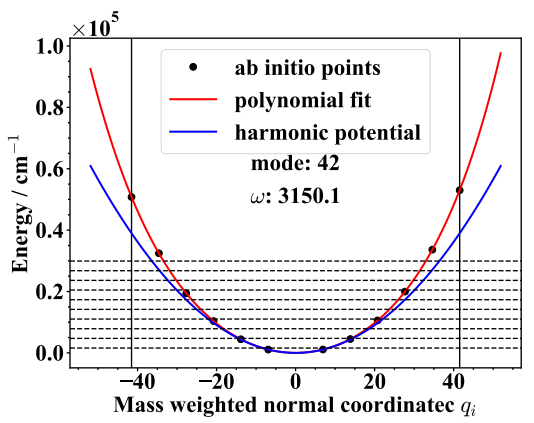

(f)

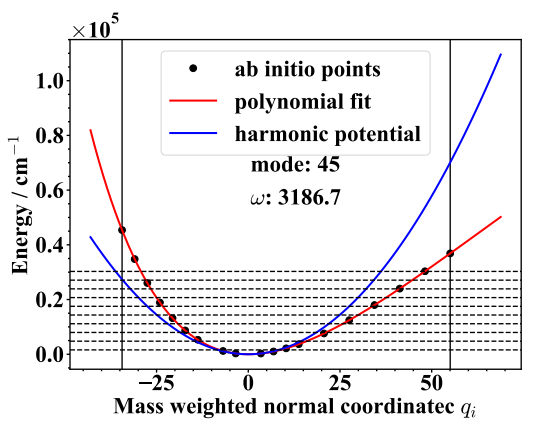

(i)

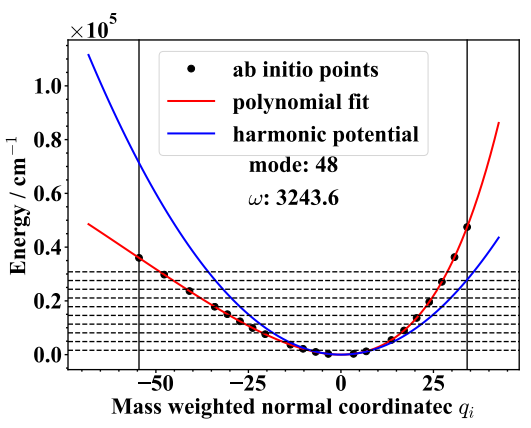

(1)

FIG. 12. Similar as Fig. 9 but for modes 37-48. 


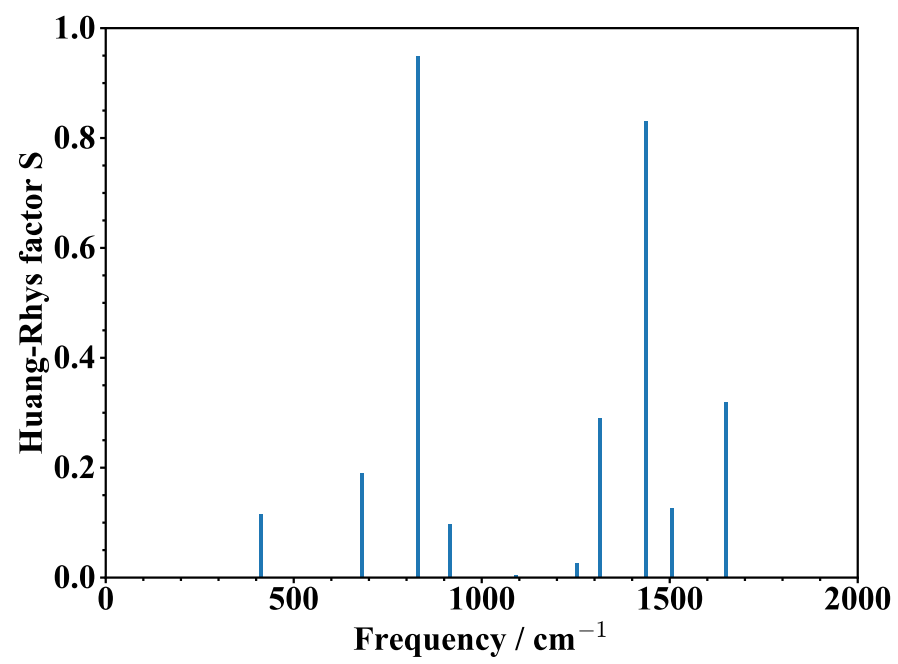

FIG. 13. The Huang-Rhys factor of different modes on the ground state PES of azulene. 